\title{
Some inverse scattering problems of geophysics
}

\author{
A G Ramm \\ Mathematics Department, Cardwell Hall, Kansas State University, Manhattan, KS 66506, \\ USA
}

Received 28 December 1984

\begin{abstract}
Several problems arising in geophysics are considered. The problems consist of finding an unknown coefficient in a partial differential equation from the knowledge of the solution to this equation on a certain surface. The unknown coefficient is usually the refraction index of a medium, the scattering potential, or the conductivity profile. Analytical inversion algorithms are given.
\end{abstract}

\section{Introduction}

There are many problems in geophysics, optics, nondestructive testing and elsewhere in which a finite inhomogeneity in a homogeneous space is to be recovered from the measurements of a field scattered by the inhomogeneity. The field can be measured far away from the inhomogeneity or in some region which is not too far from the inhomogeneity. Several problems of this type áre solved in this paper. The problems are formulated in $\S \S 2-5$. Section 2 deals with recovery of the refraction index (velocity profile) from the knowledge of the field on the plane for all positions of the source and receiver. Other problems of a similar nature are also considered. Section 3 deals with the two-parameter inversion: both the density and velocity are recovered from the knowledge of the field for all positions of the source and receiver on the plane and for two distinct frequencies. In $\S 2$ the theory is exact, in $\S 3$ it is based on the first Born approximation. In $\S 4$ the three-dimensional inverse problem of the theory of scattering by a potential is solved in the Born approximation. Section 5 deals with a model problem of induction logging theory.

The results in this paper were announced by $\operatorname{Ramm}(1983 \mathrm{a}, \mathrm{b})$. The presentation in $\S 2$ is based partly on the work of Martin and Ramm (1985), in $\S 2.5$ an idea of Blackledge (1983) is used, $\S 3$ is the work of Ramm and Weglein (1984) and $\S 5$ is the work of Ramm (1985).

\section{Inverse scattering for geophysical problems}

2.1

Let

$$
\begin{aligned}
& \nabla^{2} u+\omega^{2} n(x) u=-\delta(x-y) \quad \text { in } R^{3} \\
& n(x)=1 \quad \text { if } \quad|x| \geqslant R \quad n(x)=1+v(x) \quad \text { if } \quad|x| \leqslant R \\
& n(x)>0, \quad v(x)=0 \quad \text { if }|x| \geqslant R \quad \text { or } x_{3}>0 .
\end{aligned}
$$


The function $u$ is the outgoing (i.e. $u$ satisfies the radiation condition) scalar, e.g. acoustic, field generated by a point source located at the point $y$ in the homogeneous space with a compactly supported inhomogeneity $v(x)$ located in the lower half-space $R_{-}^{3}=\left\{x: x_{3}<0\right\}$. Let $P=\left\{x: x^{3}=0\right\}, B_{R}=\{x:|x| \leqslant R\}$. The inhomogeneity is not assumed small in any sense. No symmetry assumptions of the type $v=v\left(x_{3}\right)$ or $v=v\left(\rho, x_{3}\right)$, where $\rho=\left(x_{1}^{2}+x_{2}^{2}\right)^{1 / 2}$, are made. The only assumptions about $v(x)$ are

$$
v(x)=0, \quad|x|>R \quad \text { or } \quad x_{3}>0, \quad v(x) \in L^{2}\left(R^{3}\right),
$$

The inverse problem we are concerned with consists of finding $v(x)$ from measurements of the field $u(x, y, \omega)$ for all positions $x, y \in P$ of the receiver and source and for small $\omega$. The notion of the smallness of $\omega$ will be specified later. This is a model seismic exploration problem in which the refraction coefficient $n(x)=c^{-2}(x)$, where $c(x)$ is the wave velocity, and it is assumed that the measured field is the acoustic scalar field. One could assume that $c=c(x, \omega)$ but in this case only $c(x, 0)$ is recovered. Our method is exact and analytic. The Born approximation is not used. The assumption $v(x)=0$ if $|x| \geqslant R$ can be relaxed but fast decay of $v(x)$ at infinity is necessary for our method. For example, one can assume that

$$
|v(x)| \leqslant c\left(1+\left|x_{1}\right|^{2}+\left|x_{2}\right|^{2}\right)^{-a}\left(1+\left|x_{3}\right|\right)^{-a} \quad a>1
$$

but further relaxation of the assumptions on the rate of decay of $v$ would require some new ideas.

The requirement that $u(x, y, \omega)$ is measured for $\omega \rightarrow 0$ does not restrict the resolution ability of our method as will be clear later. Therefore the usual concept, according to which the size of the details that can be resolved by testing an object with waves of length $\lambda$ is of order $\lambda$, is not applicable to our scheme. The reason is that the data we use differ from the usual data.

There are quite a few practical examples in which the condition $\omega \rightarrow 0$ is satisfied. Currently the wavelength $\lambda$ used in seismic prospecting is in the range $50-400 \mathrm{~m}$. If the characteristic dimension $a$ of the inhomogeneity $v(x)$ satisfies the condition $a \ll \lambda$ then the condition $\omega \rightarrow 0$ is satisfied.

Practical examples include (1) metallic ore lodes $(a \sim 30 \mathrm{~m})$, (2) caves $(a \sim 20-80 \mathrm{~m})$, (3) old mine structures $(a \sim 50 \mathrm{~m})$, (4) submarines $(a \sim 50-100 \mathrm{~m})$, (5) pipelines $(a \sim 3 \mathrm{~m})$. The last problem requires some modifications in the theory because the inhomogeneity is infinite in one direction (see Ramm 1983a).

\section{2}

The method we use for solving the inverse problem is simple. One starts with the integral equation

$$
u(x, y, \omega)=g(x, y, \omega)+\omega^{2} \int g(x, z, \omega) v(z) u(z, y, \omega) \mathrm{d} z \equiv g+\omega^{2} B u
$$

where $g=\exp (\mathrm{i} \omega|x-y|) / 4 \pi|x-y|$ and the integral is taken actually over $R^{3}$ - because of (2.4). Equation (2.6) is equivalent to (2.1) which can be checked by differentiation, and the solution to (2.6) is an outgoing wave.

Under assumption (2.4) the linear integral operator $B$ in (2.6) is compact in the space $C_{y}$ of functions with a fixed singularity at the point $y$. The functions are of the form $u=C /|x-y|+h(x, y)$ where $h(x, y)$ is continuous, and the norm in this space is given by the equation $\|u\||=| C\left|+\max _{x \in R^{3}}\right| h(x, y) \mid$. 
Lemma 1. For sufficiently small $\omega$ equation (2.6) is uniquely solvable in $C_{y}$, its solution is analytic in $\omega$ in the neighbourhood of the point $\omega=0$ and can be obtained by iterations

$$
u_{n+1}=g+\omega^{2} B u_{n} \quad u_{0}=g .
$$

Proof. The conclusion of lemma 1 is an immediate consequence of the Banach fixed point theorem.

Let us rewrite (2.6) as

$$
16 \pi^{2} \frac{u-g}{\omega^{2}}=B g+\mathrm{O}\left(\omega^{2}\right)
$$

and, using lemma 1 , pass to the limit $\omega \rightarrow 0$ in (2.8). The result is

$$
M v \equiv \int \frac{v(z) \mathrm{d} z}{|x-z||z-y|}=f(x, y)
$$

where

$$
f(x, y) \equiv 16 \pi^{2} \lim _{\omega \rightarrow 0} \frac{u-g}{\omega^{2}}
$$

Let

$$
x^{1}=\left(x_{1}, x_{2}, 0\right) \quad y^{1}=\left(y_{1}, y_{2}, 0\right) .
$$

Setting $x=x^{1}$ and $y=y^{1}$ in (2.9) yields

$$
M_{\mathrm{p}} v \equiv \int \frac{v(z) \mathrm{d} z}{\left|x^{1}-z\right| y^{1}-z \mid}=f\left(x^{1}, y^{1}\right) \quad x^{1}, y^{1} \in P .
$$

This is our basic equation for finding $v(z)$ from the data $f\left(x^{1}, y^{l}\right)$.

Remark 1. One can measure the data on surfaces other than $P$. For example, on a sphere $S_{R_{1}}$ with radius $R_{1}>R$ or on a cylinder $C_{R_{1}}=\left\{x: x_{1}^{2}+x_{2}^{2}=R_{1}^{2}\right\}, R_{1}>R$. The corresponding inverse problems can be useful also. See $\S 2.5$ below.

\section{3.}

Let us solve equation (2.12). Taking the Fourier transform in $x^{1}$ and $y^{1}$ and using the formula

$$
\begin{aligned}
\frac{1}{(2 \pi)^{2}} \int_{-\infty}^{\infty} \int_{-\infty} \frac{\exp \left(\lambda \cdot x^{1}\right)}{|x-z|} \mathrm{d} x_{1} \mathrm{~d} x_{2} \\
\quad=\frac{\exp \left(\mathrm{i} \lambda \cdot z^{1}\right)}{(2 \pi)^{2}} \int_{0}^{\infty} \frac{\mathrm{d} \rho \rho}{\left(\rho^{2}+z_{3}^{2}\right)^{1 / 2}} \int_{0}^{2 \pi} \exp (\mathrm{i}|\lambda| \rho \cos \varphi) \mathrm{d} \varphi \\
\quad=\frac{\exp \left(\mathrm{i} \lambda \cdot z^{1}\right)}{2 \pi} \int_{0}^{\infty} \frac{\mathrm{d} \rho \rho J_{0}(|\lambda| \rho)}{\left(\rho^{2}+z_{3}^{2}\right)^{1 / 2}} \\
=(2 \pi)^{-1}|\lambda|^{-1} \exp \left(\mathrm{i} \lambda \cdot z^{1}\right) \exp \left(-|\lambda|\left|z_{3}\right|\right)
\end{aligned}
$$


one obtains

$$
\frac{1}{(2 \pi)^{2}} \int \mathrm{d} z v(z) \exp \left[\mathrm{i}(\lambda+\mu) \cdot z^{1}\right] \exp \left[-(|\lambda|+|\mu|)\left|z_{3}\right|\right]=|\lambda||\mu| \tilde{f}(\lambda, \mu)
$$

where

$$
\begin{array}{ll}
\lambda=\left(\lambda_{1}, \lambda_{2}\right) & |\lambda|=\left(\left|\lambda_{1}\right|^{2}+\left|\lambda_{2}\right|^{2}\right)^{1 / 2} \\
\mu=\left(\mu_{1}, \mu_{2}\right) & |\mu|=\left(\left|\mu_{1}\right|^{2}+\left|\mu_{2}\right|^{2}\right)^{1 / 2}
\end{array}
$$

and

$\tilde{f}(\lambda, \mu)=\frac{1}{(2 \pi)^{4}} \iint_{-\infty}^{\infty} \int_{-\infty}^{\cdot} \int f\left(x^{1}, y^{1}\right) \exp \left[\mathrm{i}\left(\lambda \cdot x^{1}+\mu \cdot y^{1}\right)\right] \mathrm{d} x_{1} \mathrm{~d} x_{2} \mathrm{~d} y_{1} \mathrm{~d} y_{2}$.

Let

$$
\begin{aligned}
& p=\lambda+\mu \quad p_{1}=\lambda_{1}+\mu_{1} \quad p_{2}=\lambda_{2}+\mu_{2} \\
& p_{3}=|\lambda| \quad p_{4}=|\mu| \text {. }
\end{aligned}
$$

Then (2.14) can be written as

$$
\int \mathrm{d} z v(z) \exp \left(\mathrm{i} p \cdot z^{\mathrm{l}}\right) \exp \left[-\left(p_{3}+p_{4}\right)\left|z_{3}\right|\right]=(2 \pi)^{2} p_{3} p_{4} F\left(p_{1}, p_{2}, p_{3}, p_{4}\right)
$$

where $F\left(p_{1}, p_{2}, p_{3}, p_{4}\right)$ is the function $\tilde{f}(\lambda, \mu)$ in the variables $p_{1}, p_{2}, p_{3}, p_{4}$. The Jacobian of the mapping $\left(\lambda_{1}, \lambda_{2}, \lambda_{3}, \lambda_{4}\right) \rightarrow\left(p_{1}, p_{2}, p_{3}, p_{4}\right)$ is

$$
J=\left|\begin{array}{llll}
1 & 0 & 1 & 0 \\
0 & 1 & 0 & 1 \\
\lambda_{1}|\lambda|^{-1} & \lambda_{2}|\lambda|^{-1} & 0 & 0 \\
0 & 0 & \mu_{1}|\mu|^{-1} & \mu_{2}|\mu|^{-1}
\end{array}\right|=\frac{\mu_{1} \lambda_{2}-\mu_{2} \lambda_{1}}{|\mu||\lambda|},
$$

so that $J \neq 0$ if $\mu$ and $\lambda$ are linearly independent. Let

$$
p_{3}=p_{4}=\frac{1}{2} q \text {. }
$$

Equation (2.20) determines a three-dimensional manifold in the four-dimensional space of variables $p_{1}, p_{2}, p_{3}, p_{4}$. Equation (2.18) reduces to

$$
\int_{0}^{\infty} \exp (-q \zeta)\left(\int_{-\infty}^{\infty} \int_{-\infty} \mathrm{d} z_{1} \mathrm{~d} z_{2} \exp \left(\mathrm{i} p \cdot z^{1}\right) v\left(z_{1}, z_{2},-\zeta\right)\right) \mathrm{d} \zeta=\pi^{2} \varphi\left(p_{1}, p_{2}, q\right)
$$

where

$$
\varphi\left(p_{1}, p_{2}, q\right) \equiv q^{2} F\left(p_{1}, p_{2}, \frac{1}{2} q, \frac{1}{2} q\right)
$$

and $\zeta=\left|z_{3}\right|=-z_{3}$.

Equation (21) can be solved analytically by taking the two-dimensional inverse Fourier transform and one-dimensional inverse Laplace transform:

$v\left(z_{1}, z_{2}, z_{3}\right)=\frac{1}{4} \int_{-\infty}^{\infty} \int_{-\infty}^{\infty} \mathrm{d} p_{1} \mathrm{~d} p_{2} \exp \left(-\mathrm{i} p \cdot z^{1}\right) \frac{1}{2 \pi \mathrm{i}} \int_{c-\mathrm{i} \infty}^{c+\mathrm{i} \infty} \mathrm{d} q \varphi\left(p_{1}, p_{2}, q\right) \exp \left(-q z_{3}\right)$

where $p \cdot z^{1}=p_{1} z_{1}+p_{2} z_{2}$ and $c>0$ is an arbitrary constant. The Mellin integral, which gives the inversion of the Laplace transform, does not depend on $c>0$. Computational 
aspects of the problem will be discussed in $\S 2.6$. Let us summarise the result that we have just proved.

Theorem 1. Equation (2.12) is uniquely solvable by formula (2.23) in which $\varphi$ is given in (2.22) and $F\left(p_{1}, p_{2}, p_{3}, p_{4}\right)$ is the function (2.16) in the variables (2.17).

Remark 2. The above argument does not give any explicit characterisation of the functions $f\left(x^{1}, y^{1}\right)$ for which equation (2.12) is solvable. Equation (2.12) is an integral equation of the first kind since the support of $v(z)$ is a compact set. Therefore the range of the operator $M_{P}: L^{2}\left(B_{R}\right) \rightarrow C(P \times P)$ is not closed; small perturbations of $f\left(x^{1}, y^{1}\right)$ in the norm of $C(P \times P)$ may lead to an equation which has no solution in $L^{2}\left(B_{R}\right)$, and therefore the problem of solving equation (2.12) is ill-posed. An implicit characterisation of admissible data, that is of the functions $f\left(x^{1}, y^{1}\right)$ for which equation (2.12) is solvable in $L^{2}\left(B_{R}\right)$ can be given as follows: $f\left(x^{1}, y^{1}\right)$ are admissible data iff formula (2.23) gives a function $v$ which satisfies conditions (2.4).

If the data are not measured on all of $P$ but only on a finite domain, e.g. on a disc $\mathcal{Z}_{R_{1}}$ of radius $R_{1}$, then one can still apply the inversion formula $(2.23), \tilde{f}(\lambda, \mu)$ will be the Fourier transform of the data collected on $\mathscr{V}_{R_{1}}$, but the integral (2.23) will not necessarily vanish outside $B_{R}$.

One could try a numerical method to solve equation (2.12). Since this is an equation of the first kind some regularisation is needed.

\section{4}

If the data are measured with a random error $\eta$ uniformly distributed on the interval $(-\delta, \delta), \delta>0$, then one can define the right-hand side of equation $(2.12)$ as the solution of a linear regression problem. One measures $u=u\left(x, y, \omega_{j}\right)$ at several small frequencies $\omega_{j}$,

$$
u_{j}=g+\omega_{j}^{2} b+\eta_{j} \quad 1 \leqslant j \leqslant n
$$

and estimates $b$ from these measurements. This $b$ is then taken as the right-hand side of equation (2.12). The least-squares method gives

$$
b=\left(\sum_{j=1}^{n} \omega_{j}^{4}\right)^{-1} \sum_{j=1}^{n}\left(\omega_{j}-g_{j}\right) \omega_{j}^{2}
$$

where $g_{j}=g\left(x, y, \omega_{j}\right), n$ is the number of various $\omega_{j}$ used and $b=b\left(x^{1}, y^{1}\right)$.

\section{5}

Consider the case when the lower half-space, which contains the inhomogeneity, has different properties from the upper half-space. Let $\rho_{1}, \alpha_{1}$ and $\rho_{2}, \alpha_{2}(x)$ be the density and velocity in the upper $\left(x_{3}>0\right)$ and lower $\left(x_{3}<0\right)$ half-spaces respectively. Let us assume that there is a compactly supported inhomogeneity in the lower half-space $R_{-}^{3}$, that $\rho_{1}=$ constant, $\alpha_{1}=$ constant, $\rho_{2}=$ constant, $\alpha_{2}(x)=\alpha_{2}+v(x)$, where $\alpha_{2}=$ constant and $v(x)=0$ if $|x|>R$ or $x_{3}>0$. The Green function for the problem without an inhomogeneity, i.e. for $v=0$, satisfies the equations

$$
\begin{array}{ll}
\nabla^{2} \varphi_{i}+\frac{\omega^{2}}{\alpha_{i}^{2}} \varphi_{i}=-\delta(x-y) & i=1,2 \\
\frac{\partial \varphi_{1}}{\partial x_{3}}=\frac{\partial \varphi_{2}}{\partial x_{3}} \quad \rho_{1} \varphi_{1}=\rho_{2} \varphi_{2} & \text { at } x_{3}=0 .
\end{array}
$$


Conditions (2.27) mean that the normal displacement and the pressure are continuous across the interface. $\varphi_{1}\left(\varphi_{2}\right)$ in $(2.26)-(2.27)$ denotes the value of the Green function in $\boldsymbol{R}_{+}^{3}\left(\boldsymbol{R}_{-}^{3}\right)$.

Ewing et al (1957, pp 95-6) have calculated the Green function:

$$
\varphi_{2}=\frac{1}{2 \pi} \int_{0}^{\infty} \frac{\exp \left(\nu_{2} x_{3}-\nu_{1} y_{3}\right) J_{0}(k \rho) k \mathrm{~d} k}{\delta \nu_{1}+\nu_{2}} \quad x_{3}<0
$$

where $y_{3} \geqslant 0$ (i.e. the source is in $\left.R_{+}^{3}\right), \quad \nu_{i}=\left(k^{2}-\omega^{2} \alpha_{i}^{-2}\right)^{1 / 2}, \quad \rho=\left|x^{1}-y^{1}\right|=$ $\left[\left(x_{1}-y_{1}\right)^{2}+\left(x_{2}-y_{2}\right)^{2}\right]^{1 / 2}, \delta=\rho_{2} \rho_{1}^{-1}$. Take $y_{3}=0$ to obtain

$$
\varphi_{2}=\frac{1}{2 \pi} \int_{0}^{\infty} \frac{J_{0}(k \rho) k \exp \left(\nu_{2} x_{3}\right) \mathrm{d} k}{\nu_{1} \delta+\nu_{2}} \quad x_{3}<0 .
$$

If $\omega \rightarrow 0$ then $\nu_{i} \rightarrow k$ and (2.29) takes the form

$$
\begin{aligned}
\varphi_{2} & =\frac{1}{2 \pi(\delta+1)} \int_{0}^{\infty} J_{0}(k \rho) \exp \left(-k\left|x_{3}\right|\right) \mathrm{d} k \\
& =\frac{2 \rho_{1}}{\rho_{2}+\rho_{1}} \frac{1}{4 \pi\left|x-y^{2}\right|} \quad y^{1}=\left(y_{1}, y_{2}, 0\right) .
\end{aligned}
$$

Therefore the basic integral equation (2.12) remains essentially the same:

$$
\int \frac{v(z) \mathrm{d} z}{\left|x^{1}-z\right|\left|y^{1}-z\right|}=\left(\frac{\rho_{2}+\rho_{1}}{2 \rho_{1}}\right)^{2} f\left(x^{1}, y^{1}\right)=\frac{1}{4}(1+\delta)^{2} f\left(x^{1}, y^{1}\right)
$$

where $\delta=\rho_{2} / \rho_{1}$ and $f\left(x^{1}, y^{1}\right)$ is given in (2.10) with $\varphi_{2}$ in place of $g$.

\subsection{Inversion of the data given on a sphere}

Consider equation (2.6) in the Born approximation at an arbitrary positive frequency $\omega$. This equation can be written as

$$
\int g(x, z, \omega) v(z) g(z, y, \omega) \mathrm{d} z=f(x, y) \quad x, y \in S_{R_{1}}
$$

where the right-hand side is the datum

$$
f(x, y)=(u-g) \omega^{-2}
$$

and $R_{1}>R$, so that the data are collected on the sphere $S_{R_{1}}, \int=\int_{B_{R}}$.

Let us use the well known formulae (Gradshteyn and Ryzhik 1965)

$$
\begin{aligned}
g(x, z, \omega)= & \sum_{l=0}^{\infty} C_{l} \psi_{l}^{\mathrm{re}}(z) \psi_{l}(x) \\
= & \mathrm{i} \omega \sum_{l=0}^{\infty} \sum_{m=-l}^{l} j_{l}(\omega|z|) Y_{l m}\left(z^{0}\right) h_{l}^{(\mathrm{l})}(\omega|x|) \overline{Y_{l m}\left(x^{0}\right)} \quad|x|>|z| \\
& \quad \exp (\mathrm{i} \omega n \cdot z)=\sum_{l=0}^{\infty} a_{l} \psi_{l}^{\mathrm{re}}(z) \overline{Y_{l}(n)}=4 \pi \sum_{l=0}^{\infty} \sum_{m=-l}^{l} \mathrm{i}^{l} j_{l}(\omega|z|) Y_{l m}\left(z^{0}\right) \overline{Y_{l m}(n)}
\end{aligned}
$$


where $Y_{l}$ are the normalised spherical harmonics, $\psi_{l}$ are the outgoing waves, and $\psi_{l}^{\mathrm{re}}$ are the regular waves

$$
\begin{array}{ll}
j_{l}(r)=(\pi / 2 r)^{1 / 2} J_{l+1 / 2}(r) & h_{l}^{(1)}(r)=(\pi / 2 r)^{1 / 2} H_{l+1 / 2}^{(1)}(r) \\
\psi_{l}(x)=h_{l}^{(1)}(|x|) \overline{Y_{l m}\left(x^{0}\right)} & \psi_{l}^{\mathrm{e}}(x)=j_{l}(|x|) Y_{i m}\left(x^{0}\right) \\
Y_{l m}(\theta, \varphi)=\left(\frac{2 l+1}{4 \pi} \frac{(l-m) !}{(l+m) !}\right)^{1 / 2} \mathrm{e}^{\mathrm{i} m \varphi} P_{l, m}(\cos \theta) \quad x^{0}=(\theta, \varphi) .
\end{array}
$$

Substitute (2.34) into (2.32) to obtain

$$
\sum_{l, l^{\prime}=0}^{\infty} C_{l} C_{l^{\prime}} \int v(z) \psi_{l}^{\mathrm{re}}(z) \psi_{l^{\prime}}^{\mathrm{re}}(z) \mathrm{d} z \psi_{l}(x) \psi_{l^{\prime}}(y)=\sum_{l, l^{\prime}=0}^{\infty} f_{l l^{\prime}} \overline{Y_{l}\left(x^{0}\right)} \overline{Y_{l^{\prime}}\left(y^{0}\right)}
$$

where

$$
\begin{aligned}
& f(x, y)=\sum_{l l^{\prime}=0}^{\infty} f_{l l^{\prime}} \overline{Y_{l}\left(x^{0}\right)} \overline{Y_{l^{\prime}}\left(y^{0}\right)} \\
& x^{0}=x R_{1}^{-1} \quad y^{0}=y R_{1}^{-1} .
\end{aligned}
$$

Thus the numbers $v_{l l^{\prime}}=f_{l l^{\prime}}\left(C_{l} C_{l^{\prime}} h_{l}^{(1)}\left(\omega R_{1}\right) h_{l^{\prime}}^{(1)}\left(\omega R_{1}\right)\right)^{-1}$ are known, and

$$
\int v(z) \psi_{l}^{\mathrm{re}}(z) \psi_{l^{\prime}}^{\mathrm{re}}(z) \mathrm{d} z=v_{l l}
$$

Multiply (2.37) by $a_{l} a_{l^{\prime}} \overline{Y_{l}\left(n^{\prime}\right)} \overline{Y_{l^{\prime}}(-n)}$, where $n$ and $n^{\prime}$ are arbitrary unit vectors, and use (2.35) to obtain

$$
\int v(z) \exp \left[-\mathrm{i} \omega\left(n-n^{\prime}\right) \cdot z\right] \mathrm{d} z=\sum_{l, l^{\prime}=0}^{\infty} a_{l} a_{l}, v_{l l^{\prime}} \overline{Y_{l}\left(n^{\prime}\right)} \overline{Y_{l^{\prime}}(-n)}
$$

Denote the right-hand side of $(2.38)$ by $F\left(\omega\left(n-n^{\prime}\right)\right)$ and set $\omega\left(n-n^{\prime}\right)=p$. Then

$$
\int_{B_{R}} v(z) \exp (-\mathrm{i} p \cdot z) \mathrm{d} z=F(p) \quad|p| \leqslant 2 \omega .
$$

This equation is solved in $\S 4$ (see equation (4.9)).

Let us summarise the result.

Theorem 2. Equation (2.32) has at most one solution $v \in L^{2}\left(B_{R}\right)$. If it is solvable then the solution can be computed as follows: (1) compute numbers $f_{l l}$, by formula $\left(2.36^{\prime}\right)$, (2) compute the right-hand side of (2.38), (3) solve equation (2.39) for $v(z)$ by the method of $\S 4$.

In a similar way one can solve the inverse problem in the case when $x$ and $y$ run through a cylindrical surface, $x_{1}^{2}+x_{2}^{2}=R_{1}^{2}, R_{1}>R$.

If $\omega=0$ then the above scheme should be modified because the right-hand side of (2.39) is known only at a point $p=0$ if $\omega=0$.

If $\omega=0$ then the numbers

$$
\int v(z)|z|^{l+l^{\prime}} Y_{l}\left(z^{0}\right) Y_{l^{\prime}}\left(z^{0}\right) \mathrm{d} z=v_{l l^{\prime}}^{0}
$$

are uniquely determined by the data $f(x, y)$ known for $x, y \in S_{R_{\mathrm{I}}}$. These numbers define $v(z)$ uniquely. 
Lemma 2. Equation (2.32) for $\omega=0$ has at most one solution.

Proof. Suppose $f(x, y)=0, x, y \in S_{R_{1}}$. Then the numbers $v_{l^{\prime}}^{0}=0, \forall l, l^{\prime} \geqslant 0$. We want to prove that in this case $v(z)=0$. Let

$$
v(z)=\sum_{l^{\prime \prime}=0}^{\infty} v_{l^{\prime \prime}}(r) \overline{Y_{l^{\prime \prime}}\left(z^{0}\right)} \quad 0 \leqslant r=|z| \leqslant R .
$$

Clearly, it is sufficient to prove that $v_{l}(r)=0, \forall l \geqslant 0$. It follows from $(2.40)$ and $(2.41)$ that if $v_{l \prime \prime}=0$ then

$$
\sum_{l^{\prime \prime}=0}^{\infty} \int_{0}^{R} \mathrm{~d} r r^{2+l+l^{\prime}} v_{l^{\prime \prime}}(r) b\left(l, l^{\prime}, l^{\prime \prime}\right)=0 \quad \forall l, l^{\prime} \geqslant 0
$$

where

$$
b\left(l, l^{\prime}, l^{\prime \prime}\right)=\int_{S^{2}} Y_{l}\left(z^{0}\right) Y_{l^{\prime}}\left(z^{0}\right) \bar{Y}_{l^{\prime \prime}}\left(z^{0}\right) \mathrm{d} z^{0} .
$$

Let us consider for simplicity the two-dimensional analogue of $(2.42)-(2.43)$ :

$$
\sum_{l^{\prime \prime}=-\infty}^{\infty} \int_{0}^{R} \mathrm{~d} r r^{1+|l|+\left|l^{\prime \prime}\right|} v_{l^{\prime \prime}}(r) \tilde{b}\left(l, l^{\prime}, l^{\prime \prime}\right)=0 \quad \forall l, l^{\prime}
$$

where $l, l^{\prime}, l^{\prime \prime}=0, \pm 1, \pm 2, \ldots$, and

$$
\tilde{b}\left(l, l^{\prime}, l^{\prime \prime}\right)=\frac{1}{(2 \pi)^{3 / 2}} \int_{0}^{2 \pi} \exp \left[\mathrm{i}\left(l+l^{\prime}-l^{\prime \prime}\right) \varphi\right] \mathrm{d} \varphi=\frac{1}{(2 \pi)^{1 / 2}} \delta_{0, l+l^{\prime}-l^{\prime \prime}}
$$

It follows from (2.44) and (2.45) that

$$
\int_{0}^{R} \mathrm{~d} r r^{1+|l|+\left|l^{\prime}\right|} v_{l^{\prime \prime}}(r)=0 \quad l^{\prime \prime}=l+l^{\prime}
$$

Let $l^{\prime \prime}$ be fixed. Then $1+|l|+\left|l^{\prime}\right|$ runs through all integers starting from $1+\left|l^{\prime \prime}\right|$ when $l$ and $l^{\prime}$ run through the set $0, \pm 1, \pm 2$, and $l+l^{\prime}=l^{\prime \prime}$ is fixed. Therefore

$$
\int_{0}^{R} \mathrm{~d} r r^{q} v_{l^{\prime \prime}}(r)=0 \quad q \geqslant 1+\left|l^{\prime \prime}\right|
$$

From Müntz's theorem (Akhiezer 1956) it follows that $v_{l^{\prime \prime}}(r)=0$ for all $l^{\prime \prime}$. Thus $v(z)=0$.

In the three-dimensional case one uses the formula (Lubarskii 1957, § 54)

$$
Y_{l_{1} m_{1}}(\omega) Y_{l_{2} m_{2}}(\omega)=\sum_{l^{\prime}=\mid l_{1}-l_{2} !}^{l_{1}+l_{2}} \rho\left(l_{1}, l_{2}, l^{\prime}\right)\left(l_{1} l_{2} m_{1} m_{2} \mid l^{\prime} m^{\prime}\right) Y_{l^{\prime} m^{\prime}}(\omega) \quad m^{\prime}=m_{1}+m_{2}
$$

where $\left(l_{1} l_{2} m_{1} m_{2} \mid l m\right)$ are the Clebsch-Gordan coefficients and $\rho\left(l_{1}, l_{2}, l\right)=$ $\left[\left(2 l_{1}+1\right)\left(2 l_{2}+1\right) /(2 l+1)\right]^{1 / 2}\left(l_{1} l_{2} 00 \mid l 0\right)$. Multiply the above equation by $\bar{Y}_{l_{3} m_{3}}(\omega)$ and integrate over $S^{2}$ to obtain

$$
\int_{S^{2}} Y_{l_{1} m_{1}}(\omega) Y_{l_{2} m_{2}}(\omega) \bar{Y}_{l_{3} m_{3}}(\omega) \mathrm{d} \omega=\rho\left(l_{1}, l_{2}, l_{3}\right)\left(l_{1} l_{2} m_{1} m_{2} \mid l_{3} m_{3}\right)
$$


where the formula $\int_{s^{2}} Y_{l^{\prime} m^{\prime}} \tilde{Y}_{l m} \mathrm{~d} \omega=\delta_{l l^{\prime}} \delta_{m m^{\prime}}$ was used. It follows now from $\left(2.43^{\prime}\right)$ and (2.42) that

$$
0=\sum_{l_{3}=0}^{\infty} \sum_{m_{3}=-l_{3}}^{l_{3}} \int_{0}^{R} \mathrm{~d} r r^{2+l_{1}+l_{2}} v_{l_{3} m_{3}}(r) \rho\left(l_{1}, l_{2}, l_{3}\right)\left(l_{1} l_{2} m_{1} m_{2} \mid l_{3} m_{3}\right)
$$

where we wrote $l_{1}, l_{2}$ and $l_{3}$ for $l, l^{\prime}$ and $l^{\prime \prime}$. Multiply this equation by $\left(l_{1} l_{2} m_{1}, m_{2} \mid L M\right)$, sum over $m_{1}$ and $m_{2}$, and use the orthogonality conditions (Lubarskii $1957 \S 54$ )

$$
\sum_{\substack{m_{1}, m_{2} \\ m_{1}+m_{2}=m}}\left(l_{1} l_{2} m_{1} m_{2} \mid l m\right) \overline{\left(l_{1} l_{2} m_{1} m_{2} \mid l^{\prime} m^{\prime}\right)}=\delta_{l l^{\prime}} \delta_{m m^{\prime}}
$$

to obtain

$$
0=\rho\left(l_{1}, l_{2}, L\right) \int_{0}^{R} \mathrm{~d} r r^{2+l_{1}+l_{2}} v_{L M}(r) .
$$

The coefficient $\rho\left(l_{1}, l_{2}, L\right)$ vanishes unless $l_{1}+l_{2}+L$ is even and $\left|l_{1}-l_{2}\right| \leqslant L \leqslant l_{1}+l_{2}$. Take $l_{2}=l_{1}+L$. Then the above conditions are met, and therefore $\rho\left(l_{1}, l_{1}+L, L\right) \neq 0$. Thus (2.48) yields

$$
0=\int_{0}^{R} \mathrm{~d} r r^{2+2 l_{1}+L} v_{L M}(r) \quad l_{1}=0,1,2, \ldots
$$

As above, Müntz's theorem yields $v_{L M}(r)=0$. Since $L M, L=0,1,2, \ldots,-L \leqslant M \leqslant L$, are arbitrary, $v(z)=0$.

Remark 3. The moment problem which consists of finding a function $f(r)$ from the knowledge of its moments $f_{m}$,

$$
\int_{0}^{R} f(r) r^{m} \mathrm{~d} r=f_{m} \quad m=m_{0}, m_{0}+1, m_{0}+2, \ldots,
$$

has been studied extensively (see, e.g., Akhiezer 1965).

\subsection{Computational aspects of the inversion scheme based on equation (2.21)}

(a) If one inverts the Laplace transform using the Mellin contour $\operatorname{Re} q=c$, then the function

$$
\psi\left(p_{1}, p_{2}, z_{3}\right)=(2 \pi \mathrm{i})^{-1} \int_{c-i \infty}^{c+i \infty} \varphi\left(p_{1}, p_{2}, q\right) \exp \left(-q z_{3}\right) \mathrm{d} q
$$

is computed for some values $p_{1}, p_{2}$, and $z_{3}<0$. If $q$ is a real number then, according to (2.17), $\left|p_{1}\right| \leqslant p_{3}+p_{4} \leqslant q,\left|p_{2}\right| \leqslant q$. In particular, $\left|p_{1}\right| \leqslant c$ and $\left|p_{2}\right| \leqslant c$. Therefore, for a fixed $c>0$, one obtains the values of the Fourier transform of $v\left(z_{1}, z_{2}, z_{3}\right)$ in a disc on the real two-dimensional space of the variables $p_{1}$ and $p_{2}$ :

$$
\int_{-\infty}^{\infty} \int_{-\infty} \exp \left(\mathrm{ipz} z^{1}\right) v\left(z_{1}, z_{2}, z_{3}\right) \mathrm{d} z_{1} \mathrm{~d} z_{2}=\pi^{2} \psi\left(p_{1}, p_{2}, z_{3}\right) \quad\left|p_{1}\right|^{2}+\left|p_{2}\right|^{2} \leqslant c^{2} .
$$

Of course, the function $\psi\left(p_{1}, p_{2}, z_{3}\right)$ can be computed for any desired real point $\left(p_{1}, p_{2}\right)$ by the Mellin integral provided that the parameter $c$ in this integral is chosen so that $\left|p_{1}\right| \leqslant c$ and $\left|p_{2}\right| \leqslant c$. Therefore, the function $\psi\left(p_{1}, p_{2}, z_{3}\right)$ can be computed for all real $p_{1}$ and $p_{2}$. 
However, it is of interest practically to invert the Fourier transform of a function $v$ with a known compact support (say $v=0$ for $|z|>R$ ) if the Fourier transform is known on a compact set only, for example on a disc $\left|p_{1}\right|^{2}+\left|p_{2}\right|^{2} \leqslant c^{2}$. This problem is solved in $\S 4$.

(b) Let us briefly review some methods for inverting the Laplace transform (see Bellman et al 1966, Krylov and Skoblya 1969, Davies and Martin 1979).

1. Let

$$
F(p)=L f=\int_{0}^{\infty} \exp (-p t) f(t) \mathrm{d} t \quad p=\sigma+\mathrm{i} \lambda
$$

where $|f| \leqslant c(1+t)^{-a}, a>1, c=$ constant $>0$. Then $F(p)$ is analytic in the half-plane $\sigma>0$ and continuous up to the line $\sigma=0$.

If $F(\sigma), \sigma>\sigma_{0}>0$, is given, where $\sigma_{0}$ is an arbitrary fixed number, then

$$
f(t)=\lim _{n \rightarrow \infty}\left[\frac{(-1)^{n}}{n !}\left(\frac{n}{t}\right)^{n+1} F^{(n)}\left(\frac{n}{t}\right)\right] .
$$

This is a well known formula (e.g., Davies and Martin 1979). Although higher order derivatives have appeared in (2.52) this formula can be used in practice, e.g. in the case when $F(p)$ is a rational function.

\section{Example}

$F(p)=\frac{1}{p+1} \quad f(t)=\lim _{n \rightarrow \infty}\left(\frac{(-1)^{n}}{n !} \frac{n^{n+1}}{t^{n+1}} \frac{(-1)^{n} n !}{(n / t+1)^{n+1}}\right)=\lim _{n \rightarrow \infty} \frac{n^{n+1}}{(n+t)^{n+1}}=\mathrm{e}^{-t}$.

If we can approximate $F(p)$ by a rational function $P_{\varepsilon}(p) Q_{\varepsilon}^{-1}(p)$ so that

$$
\left|F(p)-P_{\varepsilon}(p) Q_{\varepsilon}^{-1}(p)\right| \leqslant \varepsilon(1+|p|)^{-a} \quad a>1, \quad \sigma>\sigma_{0},
$$

then

$$
\max _{i \geqslant 0}\left|f(t)-f_{\varepsilon}(t)\right| \leqslant \frac{\varepsilon}{(a-1) \pi}
$$

where $f_{\varepsilon}(t)=L^{-1}\left(P_{\varepsilon}(p) Q_{\varepsilon}^{-1}(p)\right)$. The difficulty is that the simple stability estimate (2.54) holds if (2.53) holds in the complex half-plane. It would be interesting to obtain an estimate for $\left|f(t)-f_{\varepsilon}(t)\right|$ if an estimate of the type (2.53) is known on the real axis $\sigma>\sigma_{0}$ only.

2. If $F(p)$ is known in a complex half-plane $\sigma>\sigma_{0}>0$ then the Mellin inversion formula can be used:

$$
f(t)=\frac{1}{2 \pi \mathrm{i}} \int_{\sigma_{0}-\mathrm{i} \infty}^{\sigma_{0}+\mathrm{i} \infty} \exp (p t) F(p) \mathrm{d} p .
$$

Integral (2.55) can be easily reduced to the Fourier transform

$$
f(t)=\frac{1}{2 \pi} \int_{-\infty}^{\infty} \exp (\mathrm{i} \lambda t) F\left(\sigma_{0}+\mathrm{i} \lambda\right) \mathrm{d} \lambda \exp \left(\sigma_{0} t\right)
$$

which is convenient for numerical computation. There are a number of quadrature formulae and tables for numerical computation of (2.55) and the FFT routine for numerical computation of (2.56). 
3. Inversion methods which use the values of $F(p)$ on the real axis only are of particular interest. Formula (2.52) is very sensitive to the errors in $F(p)$ because it requires high-order derivatives of $F(p)$. Therefore the following methods, which are more stable towards the errors in $F(p)$, are of interest (Krylov and Skoblya 1969).

Suppose that $f$ is expanded in the Fourier-Laguerre series

$$
f(t)=\sum_{n=0}^{\infty} A_{n} \frac{\exp \left(-\frac{1}{2} t\right) L_{n}(t)}{n !}
$$

where $L_{n}(t)$ are the Laguerre polynomials

$$
L_{n}(t)=(-1)^{n}\left(t^{n}-\frac{n^{2}}{1} t^{n-1}+\frac{n^{2}(n-1)^{2}}{2 !} t^{n-2}+\ldots+(-1)^{n} n !\right) .
$$

Then

$$
A_{n}=\frac{1}{n !} \int_{0}^{\infty} \exp \left(-\frac{1}{2} t\right) L_{n}(t) f(t) \mathrm{d} t
$$

Let

$$
\alpha_{m}=\int_{0}^{\infty} \exp \left(-\frac{1}{2} t\right) t^{m} f \mathrm{~d} t
$$

Then

$$
\alpha_{m}=F^{(m)}\left(\frac{1}{2}\right)(-1)^{m}
$$

so that

$$
A_{n}=\sum_{m=0}^{n}\left(\begin{array}{l}
n \\
m
\end{array}\right) \frac{(-1)^{n-m}}{(n-m) !} \alpha_{n-m}
$$

and, by (2.57), (2.61) and (2.62),

$$
f(t)=\exp \left(-\frac{1}{2} t\right) \sum_{n=0}^{\infty} \sum_{m=0}^{n}\left(\begin{array}{l}
n \\
m
\end{array}\right) \frac{1}{(n-m) !} F^{(n-m)}\left(\frac{1}{2}\right) \frac{L_{n}(t)}{n !} .
$$

The derivatives of $F$ are present in this formula, but if the series (2.57) converges rapidly, so that only a few first terms are needed for approximating $f(t)$, then (2.63) might work better as an inversion algorithm than (2.52).

4. One can use other expansions of $f(t)$ for inverting the Laplace transform.

If $\exp (-t)=x$ then $(2.51)$ becomes

$$
\int_{0}^{1} x^{p-1} \varphi(x) \mathrm{d} x=F(p) \quad \varphi(x)=f(-\ln x)
$$

Let $p=1,2,3, \ldots$ Then

$$
\int_{0}^{1} x^{m} \varphi(x) \mathrm{d} x=F_{m} \quad m=0,1,2, \ldots
$$

This is a well known moments problem. Let $P_{n}(x)$ be the Legendre polynomials and $\tilde{P}_{n}(x)=P_{n}(2 x-1)$ be the polynomials defined on the interval $[0,1]$. 
Let

$$
f(x)=\sum_{n=0}^{\infty}(2 n+1) C_{n} \tilde{P}_{n}(x)
$$

where

$$
C_{n}=\int_{0}^{1} f(x) \tilde{P}_{n}(x) \mathrm{d} x
$$

Let

$$
\tilde{P}_{n}(y)=\sum_{m=0}^{n} P_{n m} y^{m} .
$$

It follows from (2.68) and (2.65) that

$$
C_{n}=\sum_{m=0}^{n} P_{n m} F_{m}
$$

The inversion formula is (2.66) with $C_{n}$ given by (2.69).

5. One can expand $f$ in a series of Chebyshev polynomials or a Fourier series (Krylov and Skoblya 1969). In the first case one sets $\exp (-t)=\frac{1}{2}(1+\cos \theta)$ and $f(t) \rightarrow \varphi(\theta), 0 \leqslant \theta \leqslant \pi$. In the second case one sets $\exp (-\sigma t)=\cos \theta, \sigma>0, f(t)=f\left(-\sigma^{-1} \ln \cos \theta\right) \equiv \varphi(\theta), 0 \leqslant \theta \leqslant \frac{1}{2} \pi$.

6. Finally, one can consider equation (2.51) with $p=\sigma>0$ as an equation of the first kind and solve this equation for $f(t)$ numerically. This is an ill-posed problem and therefore one should use some regularisation techniques (iterative methods, variational methods, singular value decomposition or the method of quasisolutions (e.g., Ivanov et al 1978, Tikhonov and Arsenin 1977, Ramm 1980, 1981)).

\section{8}

Consider the two-dimensional analogue of the problem (2.1)-(2.3). The data now are the values of the field $u(x, y, k)$ on the line $L=\left\{x: x_{2}=0\right\}$ for all positions of $x$ and $y$ on $L$. The assumptions about $v(x)$ are the same as in $\S 2.1$. The governing equation is equation (2.6) with

$$
g(x, y, \omega)=\frac{1}{4} \mathrm{i} H_{0}^{(1)}\left(\omega r_{x y}\right) \quad r_{x y}=|x-y|
$$

where $H_{0}^{(1)}$ is the Hankel function. The basic difference between the three-dimensional and two-dimensional problems is that the function (2.70) does not have a finite limit as $\omega \rightarrow 0$. One has

$$
g(x, y)=\alpha(\omega)+g_{0}(x, y)+\mathrm{O}\left(\omega^{2} r^{2} \log 1 / \omega\right) \quad \text { as } \omega \rightarrow 0
$$

where

$$
\alpha(\omega)=\frac{1}{2 \pi \mathrm{i}}\left(\log \frac{1}{2} \omega+\gamma-\frac{1}{2} \mathrm{i} \pi\right) \quad g_{0}=\frac{1}{2 \pi} \ln \frac{1}{r_{x y}}
$$

and $\gamma=0.5772 \ldots$ is Euler's constant. 
Let $u-g=\psi$. Equation (2.6) can be written as

$$
\psi(x, y)=\omega^{2} \int g(x, z) v(z) \psi(z, y) \mathrm{d} z+\omega^{2} \psi_{0}(x, y)
$$

where

$$
\psi_{0}(x, y)=\int g(x, z) v(z) g(z, y) \mathrm{d} z
$$

The field $\psi$ is the scattered field. Equation (2.73) is uniquely solvable in $C\left(\boldsymbol{R}^{2}\right)$ by iteration if $\omega$ is sufficiently small. It follows from (2.71)-(2.74) that

$$
\begin{gathered}
\psi(x, y)=\omega^{2}\left[\alpha^{2} \int v \mathrm{~d} z+\alpha\left(\int g_{0}(x, z) v(z) \mathrm{d} z+\int g_{0}(y, z) v(z) \mathrm{d} z\right)\right. \\
\left.+\int g_{0}(x, z) v(z) g_{0}(z, y)\right]+o\left(\omega^{2}\right) \quad \omega \rightarrow 0 .
\end{gathered}
$$

Therefore

$$
\begin{aligned}
& f_{0} \equiv \lim _{\omega \rightarrow 0}(\omega \alpha)^{-2} \psi(x, y, \omega)=\int v(z) \mathrm{d} z \equiv v_{0} \\
& f_{1} \equiv \lim _{\omega \rightarrow 0} \frac{\psi-\alpha^{2} \omega^{2} v_{0}}{\omega^{2} \alpha}=\int g_{0}(x, z) v(z) \mathrm{d} z+\int g_{0}(y, z) v(z) \mathrm{d} z
\end{aligned}
$$

and

$$
f_{2} \equiv \lim _{\omega \rightarrow 0} \frac{\psi-\alpha^{2} \omega^{2} v_{0}-\alpha \omega^{2} f_{1}}{\omega^{2}}=\int g_{0}(x, z) v(z) g_{0}(z, y) \mathrm{d} z .
$$

The quantities $f_{j}, j=0,1,2$, can be measured, and they are our data. In particular, $f_{0}$ gives the number $v_{0}$. If $x=y$ then equation (2.77) becomes

$$
\int_{\mathscr{Z}} g_{0}(x, z) v(z) \mathrm{d} z=\frac{1}{2} f_{1}(x, x) \quad x \in L, \quad \mathscr{Z}=\{x: v(x) \neq 0\}
$$

This equation is not uniquely solvable in general. Indeed, the right-hand side of (2.79) is the potential of the mass distribution $v(z)$. This potential is given on the line $L$. These data and $v_{0}$ determine the potential uniquely in the half-plane $x_{2}>0$. By the unique continuation property the potential is uniquely determined in $\Omega=R^{2} \backslash \mathscr{D}$. However, there are many distributions $v$ which produce the zero potential in $\Omega$. For example, take the origin in $\mathscr{Z}$ and let the disk $\mathscr{Z}_{r_{1}}$ belong to $\mathscr{Z}$. Choose $r_{2}$ and $r_{3}$ such that $0<r_{3}<r_{2}<r_{1}$. Define $v=\left[\pi\left(r_{2}^{2}-r_{3}^{2}\right)\right]^{-1}$ if $r_{3} \leqslant r<r_{2}, v=-\left[\pi\left(r_{1}^{2}-r_{2}^{2}\right)\right]^{-1}$ if $r_{2} \leqslant r \leqslant r_{1}, v=0$ if $r>r_{1}$ or $r<r_{3}$, $r=|x|$. Then the left-hand side of (2.79) vanishes for $r>r_{1}$, but $v \neq 0$. Under some special assumptions on $v(z)$ equation (2.79) can be uniquely solvable. For example, if $v=v\left(x_{2}\right)$ for $a \leqslant x_{1} \leqslant b$ and $-R<x_{2}<0$ and is zero otherwise, then taking the Fourier transform of (2.79) yields

$$
\int_{-\infty}^{0} v\left(z_{2}\right) \exp \left(|\lambda| z_{2}\right) \mathrm{d} z_{2}=\frac{2 \mathrm{i} \pi \lambda|\lambda| F_{1}(\lambda)}{\exp (\mathrm{i} \lambda b)-\exp (\mathrm{i} \lambda a)} \quad-\infty<\lambda<\infty
$$


where

$$
F_{1}(\lambda)=(2 \pi)^{-1} \int_{-\infty}^{\infty} f_{1}(x, x) \exp (\mathrm{i} \lambda x) \mathrm{d} x
$$

Inverting the Laplace transform (2.80) yields $v\left(z_{2}\right)$. Thus, under the above assumption about $v(z)=v\left(z_{2}\right)$ an explicit analytical inversion procedure is given in terms of the backscattered data measured on the line $x_{2}=0$.

If no special assumptions on $v$ are made then one can use equation (2.78) to recover $v$ :

$$
\begin{aligned}
& \int \log |x-z| \log |y-z| v(z) \mathrm{d} z=f(x, y) \quad x, y \in L \\
& f(x, y)=4 \pi^{2} f_{2}(x, y) .
\end{aligned}
$$

This equation is similar to (2.9). Taking the Fourier transform in $x=(x, 0)$ and $y=(y, 0)$ yields

$$
\int v(z) \exp \left[\mathrm{i}(\lambda+\mu) z_{1}-(|\lambda|+|\mu|)\left|z_{2}\right|\right] \mathrm{d} z=4|\lambda||\mu| F(\lambda, \mu)
$$

where

$$
F(\lambda, \mu)=(2 \pi)^{-2} \iint f(x, y) \exp [\mathrm{i}(\lambda x+\mu y)] \mathrm{d} x \mathrm{~d} y
$$

and

$$
\frac{1}{2 \pi} \int_{-\infty}^{\infty} \exp (\mathrm{i} \lambda x) \ln \left\{\left[\left(x-z_{1}\right)^{2}+z_{2}^{2}\right]^{1 / 2}\right\} \mathrm{d} x=-\frac{\exp \left(\mathrm{i} \lambda z_{1}-|\lambda|\left|z_{2}\right|\right)}{2|\lambda|} .
$$

Integral (2.85) can be calculated as follows. Notice that

$$
\begin{aligned}
(2 \pi)^{-1} \int_{-\infty}^{\infty} \exp (\mathrm{i} \lambda x) \ln \left\{\left[\left(x-z_{1}\right)^{2}+z_{2}^{2}\right]^{1 / 2}\right\} \mathrm{d} x \\
\quad=\exp \left(\mathrm{i} \lambda z_{1}\right)(2 \pi)^{-1} \int_{-\infty}^{\infty} \exp (\mathrm{i} \lambda y) \ln \left[\left(y^{2}+z_{2}^{2}\right)^{1 / 2}\right] \mathrm{d} y
\end{aligned}
$$

and

$$
\widehat{\ln y}=(2 \pi)^{-1} \int_{-\infty}^{\infty} \ln |y| \exp (\dot{i} \lambda y) d y=-(2|\lambda|)^{-1}
$$

Indeed,

$$
\left.\mathrm{i} \lambda \widehat{\ln |y|}=(2 \pi)^{-1} \int y^{-1} \exp (i, y) \mathrm{d} y=(2 \pi)^{-1} \mathrm{i} \pi \operatorname{sgn} \lambda=\frac{1}{2} \mathrm{i} \operatorname{sgn} \lambda\right)
$$

and $(2.85 b)$ follows. Formula (2.85) follows from $(2.85 a)$ and $(2.85 b)$ if one takes into account that integral (2.85) is a harmonic function of $\left(z_{1}, z_{2}\right)$. Another way to derive (2.85) is to differentiate the integral on the right-hand side of (2.85) with respect to $z_{2}$, calculate the resulting standard integral and integrate over $z_{2}$. Integral $(2.85 b)$ serves as an initial condition at $z_{2}=0$. 
Let

$$
p=\lambda+\mu \quad \text { and } \quad q=|\lambda|+|\mu| .
$$

As in $\S 2.3$ one can prove that equation (2.83) has at most one solution $v \in L^{2}$. The Jacobian of the mapping (2.86), $\{\lambda, \mu\} \rightarrow\{p, q\}$ is $J=\operatorname{sgn} \mu-\operatorname{sgn} \lambda \neq 0$ if $\lambda \mu<0$. Take $\mu>0$ and $\lambda<0$. Then (2.83) becomes

$$
\int v(z) \exp \left(\mathrm{i} p z_{1}+q z_{2}\right) \mathrm{d} z=\left|p^{2}-q^{2}\right| F(p, q) \quad z_{2}<0
$$

where

$$
F(p, q)=F\left[\frac{1}{2}(p-q), \frac{1}{2}(p+q)\right] .
$$

If $\lambda<0$ and $\mu>0$ then the corresponding point $(p, q)$ lies inside the wedge $0<q<\infty$, $-q<p<q$.

Equation (2.87) can be written as

$$
\int_{0}^{\infty} \exp (-q \zeta)\left(\int_{-\infty}^{\infty} v\left(z_{1},-\zeta\right) \exp \left(\mathrm{i} p z_{1}\right) \mathrm{d} z_{1}\right) \mathrm{d} \zeta=\varphi(p, q)
$$

where

$$
\varphi(p, q)=\left|p^{2}-q^{2}\right| F(p, q)
$$

Equation (2.89) is analogous to (2.21), and one can repeat the analysis given in $\S \S 2.3$ and 2.6.

\subsection{Consider the one-dimensional case}

The problem now is to find $v(x), x \in R^{1}$, from the measurements of $u(x, y, \omega)$ for $x \geqslant 0$ and $y \geqslant 0$. We assume that $v(x) \in L^{2}, v(x)=0$ if $x<-R$ or $x>-\varepsilon$ where $\varepsilon$ and $R$ are positive constants, $\varepsilon<R$. The Green function is given by

$g=\frac{\mathrm{i}}{2 \omega} \exp (\mathrm{i} \omega|x-y|) \equiv \frac{\mathrm{i}}{2 \omega}+g_{1} \equiv \frac{\mathrm{i}}{2 \omega}+g_{0}+\mathrm{O}\left(\omega r_{x y}\right) \quad$ as $\omega \rightarrow 0$

where $g_{0}=-\frac{1}{2}|x-y|, r_{x y}=|x-y|$. The integral equation (2.6) becomes

$$
u=g(x, y)+\frac{1}{2} \mathrm{i} \omega \int \exp (\mathrm{i} \omega|x-z|) v(z) u(z, y) \mathrm{d} z
$$

Let $u-g=\psi$. Then

$$
\psi(x, y)=\frac{1}{2} \mathrm{i} \omega \int \exp (\mathrm{i} \omega|x-z|) v(z) \psi(z, y) \mathrm{d} z+\psi_{0}(x, y)
$$

where

$$
\psi_{0}(x, y)=-\frac{1}{4} \int \exp (\mathrm{i} \omega|x-z|+|y-z|) v(z) \mathrm{d} z .
$$

Equation (2.93) is uniquely solvable in $C\left(\boldsymbol{R}^{1}\right)$ by iteration if $\frac{1}{2} \omega \int|v| \mathrm{d} z<1$, in particular, for sufficiently small $\omega$. Thus, $\psi$ is analytic in $\omega$ in the neighbourhood of the point $\omega=0$. The function $\psi$ is the scattered field, it is the datum. 
It follows from (2.93) that

$$
\lim _{\omega \rightarrow 0}=-\frac{1}{4} \int v \mathrm{~d} z \equiv-\frac{1}{4} v_{0} \quad v_{n} \equiv \int z^{n} v(z) \mathrm{d} z
$$

Let

$$
\psi=\psi(x, y, \omega)=\sum_{j=0}^{\infty} c_{j}(x, y)(\mathrm{i} \omega)^{j}
$$

and

$$
\begin{aligned}
& \psi_{0}=-\frac{1}{4} \sum_{j=0}^{\infty} \psi_{0 j}(\mathrm{i} \omega)^{j} \\
& \psi_{0 j}=\frac{1}{j !} \int(|x-z|+|y-z|)^{j} v(z) \mathrm{d} z .
\end{aligned}
$$

Substitute (2.96) and (2.97) into (2.93) and compare the coefficients in front of (i $\omega)^{j}$ to obtain the recurrence formula

$C_{j}(x, y)=\frac{1}{2} \sum_{m+n+1=j} \frac{1}{m !} \int|x-z|^{m} v(z) C_{n}(z, y) \mathrm{d} z-\frac{1}{4} \psi_{0 j} \quad j=0,1,2, \ldots$

If $j=0$ then (2.98) gives

$$
C_{0}(x, y)=-\frac{1}{4} \psi_{00}=-\frac{1}{4} v_{0}
$$

If $j=1$, then

$$
C_{1}(x, y)=-\frac{1}{8} v_{0}^{2}+\frac{1}{2} v_{1}-\frac{1}{4}(x+y) v_{0} .
$$

If $j=2$, then

$C_{2}(x, y)=-\frac{1}{16} v_{0}^{3}+\frac{3}{8} v_{0} v_{1}-\frac{1}{8} v_{0}^{2}(x+y)-\frac{1}{4} v_{0}^{2} x-\frac{1}{8} v_{0}(x+y)^{2}+\frac{1}{2} v_{1}(x+y)-\frac{1}{2} v_{2}$.

The coefficients $C_{j}$ can be considered as data. Equations (2.99)-(2.100) determine $v_{0}$, $v_{1}$ and $v_{2}$ recurrently. In principle all the moments $v_{n}$ (see (2.95)) can be determined. It is a classical problem, Hamburger's moment problem (Akhiezer 1965), to find $v(z)$ from the knowledge of its moments $v_{n}$ :

$$
\int_{-R}^{0} v(z) z^{n} \mathrm{~d} z=v_{n} \quad n=0,1,2, \ldots
$$

We had a similar problem in $\S 2.5$, equation (2.48). Since the system $\left\{z^{n}\right\}, n=0,1,2, \ldots$, is closed in $L^{2}(-R, 0)$, the moment problem (2.102) has at most one solution. Necessary and sufficient conditions are known for the solvability of problem (2.102) (Akhiezer 1956). A numerical solution similar to the $T$-matrix approach studied by Ramm (1982a, b) and Kristensson et al (1983) can be suggested. Namely, take a basis $\left\{\varphi_{j}(z)\right\}$ of $L^{2}(-R, 0)$, look for an approximate solution in the form

$$
v_{m}(z)=\sum_{j=0}^{m} a_{j}^{(m)} \varphi_{j}(z)
$$


substitute (2.103) into (2.102) and use the first $m$ equations (2.102) to obtain a linear system for the coefficients $a_{j}^{(m)}$ :

$$
\sum_{j=0}^{m} b_{n j} a_{j}^{(m)}=v_{n} \quad b_{n j}=\int_{-R}^{0} z^{n} \varphi_{j}(z) \mathrm{d} z
$$

We will not go into further detail because it is probably not so easy to measure higher order coefficients $C_{j}(x, y), j>2$, and therefore find the higher order moments.

It is worth mentioning that an inversion procedure within the Born approximation can be constructed easily. In the Born approximation one has $\psi \simeq \psi_{0}$, so that

$$
\int \exp (\mathrm{i} \omega|x-z|+|y-z|) v(z) \mathrm{d} z=f(x, y) \quad x, y \geqslant 0
$$

where

$$
f(x, y, \omega)=-4 \psi(x, y)
$$

is the data. Set $x=y$ in $(2.105)$ to obtain

$$
\int_{-R}^{0} \exp (-2 \mathrm{i} \omega z) v(z) \mathrm{d} z=\exp (-2 \mathrm{i} \omega x) f(x, x, \omega) \equiv f(x, \omega) .
$$

Let us fix $x \geqslant 0$ arbitrarily and consider the data $f(x, \omega)=\varphi(\omega)$ as a function of $\omega$. Then one can Fourier-invert the data $\varphi(\omega)$ and compute $v(z)$. Since $v(z)$ is compactly supported the Fourier inversion can be done also in the case when the data $\varphi(\omega)$ are given on a finite interval of frequencies, $\omega_{0} \leqslant \omega \leqslant \omega_{1}$. This is done in $\S 4$ below.

In conclusion, it is worth mentioning that if the incident field is a plane wave and the measured quantity is the reflection coefficient which is known at all frequencies, then the one-dimensional problem can be reduced by the Liouville transform to the problem of inverse scattering by a potential. This problem has been studied in the literature (e.g., Chadan and Sabatier 1977).

\subsection{Inversion of the data given on a cylinder}

Consider equation (2.32) with $x, y \in C_{R_{1}}$, where $C_{R_{1}}=\left\{x: \rho=R_{1}\right\}, R_{1}>R, \rho=\left(x_{1}^{2}+x_{2}^{2}\right)^{1 / 2}$. We have

$$
\begin{aligned}
& g(x, z)=\sum_{n=-\infty}^{\infty}(2 \pi)^{-2} \exp \left[\operatorname{in}\left(\varphi_{z}-\varphi_{x}\right)\right] \\
& \times \int_{-\infty}^{\infty} \exp \left[\mathrm{i} \hat{\lambda}\left(z_{3}-x_{3}\right)\right] \frac{1}{2} \mathrm{i} \pi J_{n}\left(\alpha \rho_{z}\right) H_{n}\left(\alpha \rho_{x}\right) \mathrm{d} \lambda \quad \rho_{z}<\rho_{x}
\end{aligned}
$$

where $\alpha=\left(k^{2}-\lambda^{2}\right)^{1 / 2}$ and $H_{n}(r)=H_{n}^{(1)}(r)$ is the Hankel function, $\rho_{x}=\rho_{y}=R_{1}, \rho_{x}=$ $\left(x_{1}^{2}+x_{2}^{2}\right)^{1 / 2}$,

$\exp \left[\mathrm{i}\left(\lambda x_{3}+\alpha \rho \cos \left(\varphi-\varphi^{\prime}\right)\right)\right]=\exp \left(\mathrm{i} \lambda x_{3}\right) \sum_{n=-\infty}^{\infty} \mathrm{i}^{n} \exp \left[\mathrm{i} n\left(\varphi-\varphi^{\prime}\right)\right] J_{n}(\alpha \rho)$ 
Equation (2.32) can be written as

$$
\begin{aligned}
-\frac{1}{64 \pi^{2}} \sum_{n=-\infty}^{\infty} & \sum_{n^{\prime}=-\infty}^{\infty} \exp \left[-\mathrm{i}\left(n \varphi_{x}+n^{\prime} \varphi_{y}\right)\right. \\
& \left.-\mathrm{i}\left(\lambda x_{3}+\lambda^{\prime} y_{3}\right)\right] H_{n}\left(\alpha R_{1}\right) H_{n^{\prime}}\left(\alpha^{\prime} R_{1}\right) \int \mathrm{d} z v(z) \exp \left[\mathrm{i}\left(n+n^{\prime}\right) \varphi_{z}\right. \\
& \left.+\mathrm{i}\left(\lambda+\lambda^{\prime}\right) z_{3}\right] J_{n}\left(\alpha \rho_{z}\right) J_{n^{\prime}}\left(\alpha^{\prime} \rho_{z}\right)=\sum_{n=-\infty}^{\infty} \sum_{n^{\prime}=-\infty}^{\infty} \int_{-\infty}^{\infty} \int_{-\infty}^{\infty} \mathrm{d} \lambda \mathrm{d} \lambda^{\prime} f_{\lambda n \lambda^{\prime} n^{\prime}} \\
& \times \exp \left[-\mathrm{i}\left(n \varphi_{x}+n^{\prime} \varphi_{y}\right)-\mathrm{i}\left(\lambda x_{3}+\lambda^{\prime} y_{3}\right)\right]
\end{aligned}
$$

where $\alpha^{\prime}=\left(k^{2}-\lambda^{\prime 2}\right)^{1 / 2}$ and

$$
\begin{gathered}
f_{\lambda n \lambda^{\prime} n^{\prime}}=\frac{1}{(2 \pi)^{4}} \int_{0}^{2 \pi} \int_{0}^{2 \pi} \int_{-\infty}^{\infty} \int_{-\infty}^{\infty} \exp \left[\mathrm{i}\left(n \varphi_{x}+n^{\prime} \varphi_{y}\right)+\mathrm{i}\left(\lambda x_{3}+\lambda^{\prime} y_{3}\right)\right] \\
\quad \times f\left(\varphi_{x}, x_{3}, \varphi_{y}, y_{3}\right) \mathrm{d} \varphi_{x} \mathrm{~d} \varphi_{y} \mathrm{~d} x_{3} \mathrm{~d} y_{3} .
\end{gathered}
$$

Here $f\left(\varphi_{x}, x_{3}, \varphi_{y}, y_{3}\right)$ is the data, the right-hand side of the equation (2.32). From (2.110) it follows that

$$
\int \mathrm{d} z v(z) \exp \left[\mathrm{i}\left(n+n^{\prime}\right) \varphi_{z}\right] J_{n}\left(\alpha \rho_{z}\right) J_{n^{\prime}}\left(\alpha^{\prime} \rho_{z}\right)=-\frac{64 \pi^{2} f_{i n \lambda^{\prime} n^{\prime}}}{H_{n}\left(\alpha R_{1}\right) H_{n^{\prime}}\left(\alpha^{\prime} R_{1}\right)} .
$$

Multiply (2.111) by i ${ }^{n+n^{\prime}} \exp \left[-\mathrm{i}\left(n \varphi+n^{\prime} \varphi^{\prime}\right)\right]$, sum over $n, n^{\prime}$ and use (2.109) to get

$$
\int_{|z| \leqslant R} \mathrm{~d} z v(z) \exp \left[\mathrm{i} k\left(m+m^{\prime}\right) \cdot z\right]=F\left[k\left(m+m^{\prime}\right)\right] \quad k>0
$$

where

$$
F\left[k\left(m+m^{\prime}\right)\right]=-64 \pi^{2} \sum_{n, n^{\prime}=-\infty}^{\infty} \frac{f_{\lambda n \lambda^{\prime} n} \mathrm{i}^{n+n^{\prime}} \exp \left[-\mathrm{i}\left(n \varphi+n^{\prime} \varphi^{\prime}\right)\right]}{H_{n}\left(\alpha R_{1}\right) H_{n}\left(\alpha^{\prime} R_{1}\right)}
$$

$m=(\sin \theta \cos \varphi, \sin \theta \sin \varphi, \cos \theta)$

$$
\begin{array}{ll}
\lambda=k \cos \theta & \alpha=k \sin \theta \\
\lambda^{\prime}=k \cos \theta^{\prime} & \alpha^{\prime}=k \sin \theta^{\prime} .
\end{array}
$$$$
m^{\prime}=\left(\sin \theta^{\prime} \cos \varphi^{\prime}, \sin \theta^{\prime} \sin \varphi^{\prime}, \cos \theta^{\prime}\right)
$$

Equation (2.112) is of the type (4.9) and can be solved as in $\S 4$.

Equation (2.9) with $x, y \in C_{R_{1}}$ is equation (2.32) with $\omega=0$. Let us prove that the homogeneous equation (2.9) with $x, y \in C_{R_{1}}$ has only the trivial solution. If

$$
w(x, y) \equiv \int_{|z| \leqslant R} \mathrm{~d} z v(z)|x-z|^{-1}|y-z|^{-1}=0 \quad x, y \in C_{R_{1}}
$$

then for any fixed $y,|y|>R$, and for $|x|>R$ the function $w(x, y)$ solves the problem

$$
\begin{array}{ll}
\nabla_{x}^{2} w(x, y)=0 & \text { if }|x| \geqslant R \text { and }|y| \geqslant R \\
w(x, y)=0 & \text { if } y \in C_{R_{1}} \text { and } x \in C_{R_{1}} ; w(\infty, y)=0 .
\end{array}
$$

Thus $w(x, y)=0$ if $|x| \geqslant R$ and $y \in C_{R_{1}}$, because (2.113) has only the trivial solution by the maximum principle. 
Fix $x,|x| \geqslant R$, and notice that $w(x, y)$ as a function of $y$ solves the probiem

$$
\begin{aligned}
& \nabla_{y}^{2} w(x, y)=0 \text { if }|x| \geqslant R \text { and }|y| \geqslant R \\
& w(x, y)=0 \quad \text { if } y \in C_{R_{1}} \text { and }|x| \geqslant R ; \quad w(x, \infty)=0 \text {. }
\end{aligned}
$$

This problem has only the trivial solution $w(x, y)=0, y_{1}^{2}+y_{2}^{2} \geqslant R_{1}^{2},|x| \geqslant R$. Therefore, by the unique continuation property for solutions to Laplace's equation, $w(x, y)=0$ if $|x| \geqslant R$ and $|y| \geqslant R$. In particular, $w(x, y)=0$ if $x, y \in P$, where $P$ is a plane which does not intersect the support of $v$. This implies that $v=0$ as is proved in theorem 1 .

\section{Two-parameter inversion}

\section{1}

In $\S 2$, one unknown function $n(x)$ was found from the seismic data. In this section we use the data at two frequencies to find the density of the inhomogeneity and the velocity. Consider the equation

$$
\frac{\omega^{2} G}{k(x)}+\nabla \cdot\left(\frac{1}{\rho(x)} \nabla G\right)=-\delta(x-y)
$$

where $x, y \in R^{3}, \rho(x)$ is the density and $k(x)$ is the bulk modulus. Let us assume that

$$
\frac{1}{k(x)}=\frac{1}{k_{r}}-\frac{a_{1}(x)}{\rho_{r}} \quad \frac{1}{\rho}=\frac{1}{\rho_{r}}-\frac{a_{2}(x)}{\rho_{r}}
$$

where $k_{r}$ and $\rho_{r}$ are positive constants, and $a_{j}(x), j=1,2$, are functions with compact support, i.e. $a_{j}(x)=0$ if $|x|>R$, where $R>0$ is an arbitrarily large fixed number. We assume that $a_{1} \in L^{2}\left(B_{R}\right), B_{R}=\{x:|x| \leqslant R\}$ and $a_{2} \in H^{1}\left(B_{R}\right)$.

Equation (3.1) can be written as

$$
\left(\nabla^{2}+\frac{\omega^{2}}{c^{2}}\right) G-\omega^{2} a_{1} G-\nabla \cdot\left(a_{2} \nabla G\right)=-\rho_{r} \delta(x-y)
$$

where $c^{2}=k_{r} \rho_{r}^{-1}$. Let us choose units in which

$$
c=1 \quad \rho_{r}=1 \text {. }
$$

This is possible: take $x=\alpha x^{\prime}, y=\alpha y^{\prime}, \omega=\beta \omega^{\prime}$ where $\alpha$ and $\beta$ are constants. Then (3.3) takes the form

$\left.\left(\alpha^{-2} \nabla^{\prime 2}+\beta^{2} \omega^{\prime 2} / c^{2}\right) G-\beta^{2} \omega^{2} a_{1} G-\alpha^{-2} \nabla^{\prime} \cdot a_{2} \nabla^{\prime} G\right)=-\rho_{r}^{-3} \alpha \delta\left(x^{\prime}-y^{\prime}\right)$.

Let $\alpha=\rho_{r}, \beta=c \rho_{r}^{-1}$ and define $a_{1}^{\prime}=c^{2} a_{1}$ and $a_{2}^{\prime}=a_{2}$. Then (3.5) takes the form

$$
\left(\nabla^{\prime 2}+\omega^{\prime 2}\right) G-\omega^{2} a_{1}^{\prime}(x) G-\nabla^{\prime} \cdot\left(a_{2}^{\prime} \nabla G\right)=-\delta\left(x^{\prime}-y^{\prime}\right) .
$$

Therefore one can study the equation

$$
\left.\left(\nabla^{2}+\omega^{2}\right) G-\omega^{2} a_{1}(x) G-\nabla \cdot a_{2} \nabla G\right)=-\delta(x-y) .
$$

This equation can be written as the integral equation (which is analogous to equation (2.6))

$$
G=g-\omega^{2} \int g a_{1} G \mathrm{~d} z-\int g \nabla_{z} \cdot\left(a_{2}(z) \nabla_{z} G\right) \mathrm{d} z
$$


where $\int=\int_{R^{3}}, \mathrm{~d} z=\mathrm{d} z_{1} \mathrm{~d} z_{2} \mathrm{~d} z_{3}$ and

$$
g=\exp (\mathrm{i} \omega|x-y|) / 4 \pi|x-y| \text {. }
$$

In operator form equation (3.8) can be written as

$$
G=g-\omega^{2} g a_{1} G-g \nabla \cdot\left(a_{2} \nabla G\right)=g-g V G
$$

where the potential $V$ is given by

$$
V=\omega^{2} a_{1}+\nabla \cdot a_{2} \nabla
$$

Notice that $G=g-G V g$ so that $G V g=g V G$. Let us define the $T$ matrix

$$
T=V-V G V \text {. }
$$

The $T$ matrix here is not to be confused with the $T$ matrix of Ramm (1982a, b, 1983c). It is easy to check that

$$
T=V-V g T
$$

and

$$
T g=V G
$$

The operator $V$ is an integral operator in impulse space with kernel

$$
(2 \pi)^{-3} \tilde{V}(k, p)=(2 \pi)^{-3} \iint \mathrm{d} x \mathrm{~d} y V(x, y) \exp (\mathrm{i} k \cdot x-p \cdot y)
$$

where $V(x, y)$ is the kernel of $V$. This kernel may be a distribution. For $V$ defined in (3.11) we have $V(x, y)=\omega^{2} a_{1}(x) \delta(x-y)+\nabla \cdot a_{2}(y) \nabla \delta(x-y)$, so that

$$
\tilde{V}(k, p)=\omega^{2} \tilde{a}_{1}(k-p)-k \cdot p \tilde{a}_{2}(k-p)
$$

where $\tilde{a}(k)=\int \exp (\mathrm{i} k \cdot x) a(x) \mathrm{d} x$. The scattered field $G_{\mathrm{s}}=G-g$ according to equations (3.10) and (3.14) can be written as

$$
G_{s}=-g V G=-g T g \text {. }
$$

Let us keep the variables $x$ and $y$ (the positions of the receiver and the source) on the plane $x_{3}=0$ and Fourier transform (3.16) in $\hat{x}=\left(x_{1}, x_{2}\right)$ and $\hat{y}=\left(y_{1}, y_{2}\right)$. Let $\hat{k} \cdot x=k_{1} x_{1}+k_{2} x_{2}$, $\mathrm{d} \hat{x}=\mathrm{d} x_{1} \mathrm{~d} x_{2}$, etc. Then

$$
\begin{aligned}
\tilde{G}_{s}(\hat{k}, \hat{p}, \omega)= & \int \exp (i \hat{k} \cdot \hat{x}-\mathrm{i} \hat{p} \cdot \hat{y}) G_{\mathrm{s}}(\hat{x}, \hat{y}, \omega) \mathrm{d} \hat{x} \mathrm{~d} \hat{y} \\
= & -\int \exp (\mathrm{i} \hat{k} \cdot \hat{x}-\mathrm{i} \hat{p} \cdot \hat{y}) g T g \mathrm{~d} \hat{x} \mathrm{~d} \hat{y} \\
= & -\iint \mathrm{d} z^{\prime} \mathrm{d} z^{\prime \prime} T\left(z^{\prime}, z^{\prime \prime}\right) \int \frac{\exp \left(\mathrm{i} \omega\left|z^{\prime}-\hat{x}\right|+\mathrm{i} \hat{k} \hat{x}\right)}{4 \pi\left|z^{\prime}-\hat{x}\right|} \mathrm{d} \hat{x} \\
& \times \int \frac{\exp \left(\mathrm{i} \omega\left|z^{\prime}-\hat{y}\right|-\mathrm{i} \hat{p} \cdot \hat{y}\right)}{4 \pi\left|z^{\prime \prime}-\hat{y}\right|} \mathrm{d} \hat{y} \\
= & -\iint \mathrm{d} z^{\prime} \mathrm{d} z^{\prime \prime} T\left(z^{\prime}, z^{\prime \prime}\right) \exp \left(\hat{\mathrm{i}} \hat{k} \hat{z}^{\prime}-\mathrm{i} \hat{p} \cdot \hat{z}^{\prime \prime}\right) \\
& \times h\left(\left|z_{\hat{3}}^{\prime}\right|,|\hat{k}|\right) \cdot h\left(\left|z^{\prime \prime}\right|,|\hat{p}|\right)
\end{aligned}
$$


where $T\left(z^{\prime}, z^{\prime \prime}\right)$ is the kernel of $T$,

$$
h\left(\left|z_{3}^{\prime}\right|,|\hat{k}|\right)= \begin{cases}\frac{i}{2} \frac{\exp \left[i\left|z_{3}^{\prime}\right|\left(\omega^{2}-|\hat{k}|^{2}\right)^{1 / 2}\right]}{\left(\omega^{2}-|\hat{k}|^{2}\right)^{1 / 2}} & \omega>|\hat{k}| \\ \frac{1}{2} \frac{\exp \left[-\left|z_{3}^{\prime}\right|\left(|\hat{k}|^{2}-\omega^{2}\right)^{1 / 2}\right]}{\left(|\hat{k}|^{2}-\omega^{2}\right)^{1 / 2}} & \omega<|\hat{k}| \\ \infty & \omega=|\hat{k}| .\end{cases}
$$

Let us give the details of the computation of integral (18). One has, for example,

$$
\begin{aligned}
&\left.\int \frac{\exp (\hat{\mathrm{k}} \cdot x+\mathrm{i} \omega|z-\hat{x}|)}{4 \pi|\hat{x}-z|} \mathrm{d} \hat{x}\right|_{\hat{x}=\hat{u}+\hat{z}} \\
&=\exp (\mathrm{i} \hat{k} \cdot \hat{z}) \int \frac{\exp \left[\mathrm{i}|\hat{k}||\hat{u}| \cos \varphi+\mathrm{i} \omega\left(|\hat{u}|^{2}+z_{3}^{2}\right)^{1 / 2}\right]}{4 \pi\left(|\hat{u}|^{2}+z_{3}^{2}\right)^{1 / 2}} \mathrm{~d} \hat{u} \\
&= \frac{\exp (\mathrm{i} \hat{k} \cdot \hat{z})}{4 \pi} \int_{0}^{\infty} \frac{\mathrm{d} \rho \rho \exp \left[\mathrm{i} \omega\left(\rho^{2}+z_{3}^{2}\right)^{1 / 2}\right]}{\left(\rho^{2}+z_{3}^{2}\right)^{1 / 2}} \int_{0}^{2 \pi} \mathrm{d} \varphi \exp (\mathrm{i}|\hat{k}| \rho \cos \varphi) \\
&= \frac{\exp (\mathrm{i} \hat{k} \cdot \hat{z})}{4 \pi} \frac{2 \pi}{\mid \hat{k}^{1 / 2}} \int_{0}^{\infty} \mathrm{d} \rho \rho^{1 / 2} \exp \left[\mathrm{i} \omega\left(\rho^{2}+z_{3}^{2}\right)^{1 / 2} \sqrt{\rho|\hat{k}|} J_{0}(\rho|\hat{k}|)\right. \\
&= \frac{\exp (\mathrm{i} \hat{k} \cdot \hat{z})}{2|\hat{k}|^{1 / 2}} \chi
\end{aligned}
$$

where (see Erdelyi et al 1954, formula (8.6.21))

$$
\chi= \begin{cases}\mathrm{i}\left(\frac{1}{2} \pi\left|z_{3}\right|\right)^{1 / 2}|\hat{k}|^{1 / 2} \frac{H_{-1 / 2}^{(1)}\left[\left|z_{3}\right|\left(\omega^{2}-|\hat{k}|^{2}\right)^{1 / 2}\right]}{\left(\omega^{2}-|\hat{k}|^{2}\right)^{1 / 4}} & \omega>|\hat{k}| \\ \left(\frac{2\left|z_{3}\right|}{\pi}\right)^{1 / 2}|\hat{k}|^{1 / 2} \frac{K_{1 / 2}\left[\left|z_{3}\right|\left(|\hat{k}|^{2}-\omega^{2}\right)^{1 / 2}\right]}{\left(|\hat{k}|^{2}-\omega^{2}\right)^{1 / 4}} & \omega<|\hat{k}|\end{cases}
$$

and

$$
H_{-1 / 2}^{(1)}(z)=(2 / \pi z)^{1 / 2} \exp (\mathrm{i} z) \quad K_{1 / 2}(z)=(\pi / 2 z)^{1 / 2} \exp (-\mathrm{z}) .
$$

Formula (3.18) follows from the last three formulae. Let us define

$$
k_{3}= \begin{cases}\left(\omega^{2}-|\hat{k}|^{2}\right)^{1 / 2} & |\hat{k}|<\omega \\ \mathrm{i}\left(\left.\hat{k}\right|^{2}-\omega^{2}\right)^{1 / 2} & |\hat{k}|>\omega\end{cases}
$$

and $p_{3}$ in the same way. Then

$$
|\hat{k}|^{2}+k_{3}^{2}=\omega^{2} \quad p_{3}^{2}+|\hat{p}|^{2}=\omega^{2} .
$$

The vectors $\hat{k}$ and $\hat{p}$ are real valued but $k_{3}$ and $p_{3}$ may be complex numbers. If $V$ has compact support (and this was our assumption) then $T$ has compact support as follows from (3.12). Therefore the Fourier transform of $T$ is meaningful for complex $p_{3}$ and $k_{3}$. 
From (3.17) and (3.19) one obtains

$\tilde{G}_{\mathrm{s}}=\frac{1}{4 k_{3} p_{3}} \iint \mathrm{d} z^{\prime} \mathrm{d} z^{\prime \prime} T\left(z^{\prime}, z^{\prime \prime}\right) \exp \left(\mathrm{i} \hat{k} \cdot \hat{z}^{\prime}+\mathrm{i} k_{3}\left|z_{3}^{\prime}\right|-\mathrm{i} \hat{p} \cdot \hat{z}^{\prime \prime}+\mathrm{i} p_{3}\left|z_{3}^{\prime \prime}\right|\right)$

Let us assume that the support of $V$ belongs to the half-space $z_{3}<0$. Then $\left|z_{3}\right|=-z_{3}$ and (3.21) takes the form

$$
\begin{array}{ll}
\tilde{G}_{\mathrm{s}}=\frac{1}{4 k_{3} p_{3}} \tilde{T}\left(k^{\prime}, p\right) & |k|=|p|=\omega \\
k^{\prime} \equiv\left(\hat{k},-k_{3}\right) & \left|k^{\prime}\right|=|k|
\end{array}
$$

and

$$
\tilde{T}(k, p) \equiv \iint \mathrm{d} z^{\prime} \mathrm{d} z^{\prime \prime} T\left(z^{\prime}, z^{\prime \prime}\right) \exp \left(\mathrm{i} k \cdot z^{\prime}-p \cdot z^{\prime \prime}\right)
$$

The basic problem can be formulated as follows. Given the scattering data $G\left(\hat{x}, x_{3}=\right.$ $\left.0, \hat{y}, y_{3}=0, \omega\right)$ for all $\hat{x}$ and $\hat{y}$ and two distinct frequencies $\omega_{j}, j=1,2$, or small $\omega$, find $a_{j}(x), j=1,2$.

Formulae (3.12), (3.15) and (3.22) are basic for our first inversion scheme with the data given at two distinct frequencies. Our second inversion scheme is based on the method given in $\S 2$.

The inversion schemes can be generalised to include the dissipative terms (corresponding to first derivative in time with a coefficient depending on $x$ but not on $t$ in the time-dependent problem). Our method is based on the Born approximation. Since the potential (3.11) contains second derivatives it does not become small even for $\omega \rightarrow 0$.

\section{2}

Let us describe the first inversion scheme. Formula (3.15) shows that under the assumption

$$
|k|=|p|=\omega,
$$

which means that the $T$ matrix in (3.22) is known on shell, $\tilde{V}$ can be written as

$$
\tilde{V}(k, \vec{p})=\omega^{2} W(k, p) \quad W=\tilde{a}_{1}(k-p)-k^{0} \cdot p^{0} \tilde{a}_{2}(k-p)
$$

where $k^{0}=k|k|^{-1}$ and $p^{0}=p|p|^{-1}$ are unit vectors which do not depend on $\omega$. Since the functions $a_{1}$ and $a_{2}$ have compact support their Fourier transforms $\tilde{a}_{1}$ and $\tilde{a}_{2}$ are entire functions of the three complex variables $k_{1}, k_{2}$ and $k_{3}$, which decay at infinity in the real space in $\boldsymbol{R}^{3}$.

In the Born approximation $T=V$ because one neglects the quadratic terms in $V$ in (3.14), and in this approximation (3.22) becomes

$$
\tilde{G}_{\mathrm{s}}=\frac{1}{4 k_{3} p_{3}} \tilde{V}\left(k^{\prime}, p\right)=\frac{\omega^{2}}{4 k_{3} p_{3}} W\left(k^{\prime}, p\right) .
$$

Thus

$$
4 \tilde{G}_{s} k_{3} p_{3} \omega^{-2}=W\left(k^{\prime}, p\right)
$$


Let

$$
\begin{array}{ll}
k^{\prime}-p=q & k^{\prime}+p=s \\
k^{\prime}=\frac{1}{2}(s+q) & p=\frac{1}{2}(s-q) .
\end{array}
$$

Then

$$
\begin{aligned}
& k^{\prime} \cdot p=\frac{1}{4}\left(|s|^{2}-|q|^{2}\right) \quad s \cdot q=0 \\
& k^{\prime 0} \cdot p^{0}=\left(|s|^{2}-|q|^{2}\right)\left(4 \omega^{2}\right)^{-1} \\
& |q|^{2}=2 \omega^{2}\left(1-k^{\prime 0} \cdot p^{0}\right) \\
& |s|^{2}=\left|k^{\prime}\right|^{2}+|p|^{2}+2 k^{\prime} \cdot p=2 \omega^{2}\left(1+k^{\prime 0} \cdot p^{0}\right) \quad \omega>0 \\
& |s|^{2}-|q|^{2}=4 \omega^{2}-2|q|^{2} .
\end{aligned}
$$

Therefore

$$
W \equiv W\left(k^{\prime}, p\right)=\tilde{a}_{1}(q)-\tilde{a}_{2}(q) \frac{|s|^{2}-|q|^{2}}{4 \omega^{2}}=\tilde{a}_{1}(q)-\tilde{a}_{2}(q)\left(1-\frac{|q|^{2}}{2 \omega^{2}}\right) \quad \omega>0 .
$$

Let us take two arbitrary frequencies $\omega_{1}$ and $\omega_{2} \neq \omega_{1}$ and solve the two equations

$$
\begin{aligned}
& W_{1}=\tilde{a}_{1}(q)-\tilde{a}_{2}(q)\left(1-|q|^{2} / 2 \omega_{1}^{2}\right) \\
& W_{2}=\tilde{a}_{1}(q)-\tilde{a}_{2}(q)\left(1-|q|^{2} / 2 \omega_{2}^{2}\right)
\end{aligned}
$$

for $\tilde{a}_{1}$ and $\tilde{a}_{2}(q)$. The functions $W_{j}, j=1,2$, are the data according to (3.27). One obtains

$$
\begin{aligned}
& \tilde{a}_{2}=\frac{2\left(W_{1}-W_{2}\right)}{|q|^{2}} \frac{\omega_{1}^{2} \omega_{2}^{2}}{\omega_{2}^{2}-\omega_{1}^{2}} \\
& \tilde{a}_{1}=W_{1}+\left(W_{1}-W_{2}\right) \frac{2 \omega_{1}^{2} \omega_{2}^{2}}{\left(\omega_{2}^{2}-\omega_{1}^{2}\right)|q|^{2}}\left(1-\frac{|q|^{2}}{2 \omega_{1}^{2}}\right) .
\end{aligned}
$$

Taking the inverse Fourier transform of the functions (3.37) and (3.38) one obtains $a_{2}(x)$ and $a_{1}(x)$. It is clear from (3.34) that the following conclusion holds.

Conclusion. One cannot find both functions $a_{1}$ and $a_{2}$ if the data are given at only one frequency. Indeed, it follows from (3.34) that for a fixed frequency the data depends on $q$ only, and there is no parameter to vary in order to find both $\tilde{a}_{1}$ and $\tilde{a}_{2}$. The conclusion that one cannot recover both functions $a_{j}, j=1,2$, from the data given at one frequency is not at all obvious: at first glance one can think that the two conditions $|k|=|p|=\omega$ leave four degrees of freedom in the six-dimensional space $\boldsymbol{R}_{k}^{3} \times \boldsymbol{R}_{p}^{3}$, which should be enough to determine two functions $\tilde{a}_{j}, j=1,2$, of three variables. This argument, however, is not valid as one saw above. The reason is that the function $W\left(k^{\prime}, p\right)$ has a very special structure as a function of two vectors $k^{\prime}$ and $p$. Our reasoning applies to the theory in the Born approximation. It does not imply that the conclusion holds in exact theory. The exact theory unfortunately is out of reach.

Another important point concerns the numerical problem of inverting the Fourier transforms of $\tilde{a}_{j}, j=1,2$. Let $\omega_{1}<\omega_{2}$. It follows from (3.32) that the real-valued vectors $q$ run through the ball $B_{2 \omega}=\{q:|q| \leqslant 2 \omega\}$ when $k^{\prime 0}$ and $p^{0}$ run through the unit sphere $S^{2}$, so that the number $k^{\prime 0} \cdot p^{0}$ runs through the interval $[-1,1]$. Therefore, for real-valued vectors $q$ formulae (3.37) and (3.38) determine the Fourier transforms $\tilde{a}_{j}, j=1,2$, in the ball $B_{2 \omega_{1}}$ only. Since $a_{j}(x)$ are compactly supported the functions $\tilde{a}_{j}(q)$ are entire analytic 
functions of $q$. Therefore if one knows these functions in a ball $B_{2 \omega_{1}}$, one can uniquely determine these functions everywhere in $R^{3}$ by analytic continuation. However, the basic numerical problem is as follows.

Problem. Given the Fourier transform $\tilde{a}(q)$ of a function $a(x), a(x)=0$ if $|x| \geqslant R$, in the ball $B_{2 \omega}=\{q,|q|<2 \omega\}$, compute $a(x)$ in the ball $B_{R}=\{x:|x| \leqslant R\}$ with a prescribed accuracy.

This problem will be treated in $\S 4$.

\section{3}

Let us describe the second inversion scheme. If $\omega \rightarrow 0$ the limit of equation (3.8) is

$$
G(x, y)=g_{0}(x, y)-\int g_{0} \nabla \cdot\left(a_{2} \nabla G\right) \mathrm{d} z \quad g_{0}=\frac{1}{4 \pi|x-y|} .
$$

The scattered field $G_{s}=G-g_{0}$ in the Born approximation for $x$ and $y$ on the plane $x_{3}=0$ is of the form

$$
G_{s}(\hat{x}, \hat{y})=-\frac{1}{16 \pi^{2}} \int \frac{1}{|\hat{x}-z|} \nabla \cdot\left(a_{2}(z) \nabla \frac{1}{|z-\hat{y}|}\right) \mathrm{d} z .
$$

This equation can be solved analytically by the method given in $\S 2$. One takes the Fourier transform in $\hat{x}$ and $\hat{y}$ of equation (3.40), uses formula (2.13) and obtains

$$
\begin{aligned}
f(\lambda, \mu) & \equiv \frac{1}{(2 \pi)^{4}} \iint \mathrm{d} \hat{x} \mathrm{~d} \hat{y} \exp (\mathrm{i} \lambda \cdot \hat{x}+\mathrm{i} \mu \cdot \hat{y})\left(-16 \pi^{2} G_{\mathrm{s}}(\hat{x}, \hat{y})\right) \\
& =\frac{1}{(2 \pi)^{2}} \int \mathrm{d} z \frac{\exp \left(\mathrm{i} \lambda \cdot \hat{z}-|\lambda|\left|z_{3}\right|\right)}{|\lambda|} \nabla_{z} \cdot\left(a_{2}(z) \nabla_{z} \frac{\exp \left(\mathrm{i} \mu \cdot \hat{z}-|\mu|\left|z_{3}\right|\right)}{|\mu|}\right) \\
& =\frac{-1}{(2 \pi)^{2}|\lambda||\mu|} \int \mathrm{d} z \exp \left[\mathrm{i}(\lambda+\mu) \cdot \hat{z}+(|\lambda|+|\mu|) z_{3}\right] a_{2}(z)\left(\mathrm{i} \lambda+|\lambda| e_{3}\right) \\
\left(\mathrm{i} \mu+|\mu| e_{3}\right) & =\frac{(\lambda \cdot \mu-|\lambda||\mu|)}{(2 \pi)^{2}|\lambda||\mu|} \int \mathrm{d} z a_{2}(z) \exp \left(\mathrm{i} p \cdot \hat{z}+q \cdot z_{3}\right) .
\end{aligned}
$$

Here we integrated once by parts, used the equality $\left|z_{3}\right|=-z_{3}$ and set $p=\lambda+\mu, q=$ $|\lambda|+|\mu|,\left(p_{1}, p_{2}\right), p_{3}=|\lambda|, p_{4}=|\mu|$. These $p$ and $q$ should not be confused with $p$ and $q$ in $\S 3.2$. They are the same as in $\S 2$.

Define

$$
\begin{aligned}
F(p, q) & \equiv \frac{(2 \pi)^{2}|\lambda||\mu| f(\lambda, \mu)}{\lambda \cdot \mu-|\lambda||\mu|}=\int \mathrm{d} z a_{2}(z) \exp \left(\mathrm{i} p \cdot \hat{z}+q \cdot z_{3}\right) \\
& =\int_{-\infty}^{\infty} \int_{-\infty} \mathrm{d} p \exp (\mathrm{i} p \cdot \hat{z}) \int_{0}^{\infty} \mathrm{d} \zeta a_{2}(\hat{z},-\zeta) \exp (-q \zeta)
\end{aligned}
$$

as in $\S 2$. Here $\zeta=-z_{3}$. The function $F(p, q)$ is the datum. Taking the inverse Fourier and Laplace transforms of $F(p, q)$ one finds $a_{2}(z)=a_{2}(\hat{z},-\zeta)$ :

$a_{2}(z)=\frac{1}{(2 \pi)^{2}} \int_{-\infty}^{\infty} \int_{-\infty} \mathrm{d} p \exp (-\mathrm{i} p \cdot z) \frac{1}{2 \pi \mathrm{i}} \int_{c-i \infty}^{c+i \infty} \mathrm{d} q F(p, q) \exp \left(-q z_{3}\right) \quad c>0$. 
If $a_{2}(z)$ is found then equation (3.8) in the Born approximation and for $x=(\hat{x}, 0), y=(\hat{y}, 0)$ can be written as

$$
-16 \pi^{2} \lim _{\omega \rightarrow 0} \frac{G_{s}-Q}{\omega^{2}}=\int \frac{a_{1}(z) \mathrm{d} z}{|\hat{x}-z||\hat{y}-z|}
$$

where

$$
Q=-\int g(\hat{x}, z) \nabla_{z} \cdot\left(a_{2}(z) \nabla_{z} g(z, \hat{y})\right) \mathrm{d} z
$$

and $a_{2}(z)$ is given by (3.43).

Equation (3.44) was solved in $\S 2$. Let us summarise the second inversion scheme. First, one computes $a_{2}$ by formula (3.43), then $a_{1}$ by solving equation (3.44).

\section{4}

In this section we give sufficient conditions for convergence of the Born series. These conditions can be considered as conditions under which the Born approximation is valid. Error estimates will be obtained.

Consider the equation

$$
u=f+\omega^{2} T_{1} u+T_{2} u
$$

where

$$
\begin{aligned}
& T_{1} u=\int g a_{1} u \mathrm{~d} z \\
& T_{2} u=\int g \nabla \cdot\left(a_{2}(z) \nabla u\right) \mathrm{d} z .
\end{aligned}
$$

The assumptions about $a_{j}(x)$ are

$$
\begin{array}{ll}
a_{j}(x)=0 & \text { if }|x| \geqslant R \\
\left|a_{1}\right| \leqslant c_{1} & \left|a_{2}\right|+\left|\nabla a_{2}\right| \leqslant c_{2} .
\end{array}
$$

The Born series for equation (3.47) is of the form $u=\sum_{n=0}^{\infty}\left(\omega^{2} T_{1}+T_{2}\right)^{n} f$. It converges in some space $H$ if $\left\|\omega^{2} T_{1}+T_{2}\right\|<1$ where $\|T\|$ is the norm of a linear operator $T$ acting in this space. Let $H=H^{2}\left(B_{R}\right)$ be the Sobolev space, $B_{R}=\{x:|x| \leqslant R\}$. Since the functions $a_{j}(x)$ vanish outside $B_{R}$ the values of the functions $T_{j} f$ are determined entirely by the values of $f$ on $B_{R}$. The estimate

$$
\|g f\|_{2, R} \leqslant c(R)\|f\|_{0, R}
$$

is known (see, e.g., Kantorovich and Akilov 1980). Here $\|u\|_{m, R}=\|u\|_{H^{m}\left(B_{R}\right)}$. If $\omega \rightarrow 0$ the inequality $\left\|\omega^{2} T_{1}+T_{2}\right\|<1$ holds if $\left\|T_{1}\right\| \leqslant c$ and $\left\|T_{2}\right\|<1$. Here and below we denote various constants by $c$. One has, using (3.49),

$$
\left\|T_{1} f\right\|_{2, R} \leqslant c_{R}\left\|a_{1} f\right\|_{0, R} \leqslant c_{R} c_{1}\|f\|_{0, R} \leqslant c\|f\|_{2, R} .
$$

Thus $\left\|T_{1}\right\| \leqslant c$ if $\left|a_{1}\right| \leqslant c_{1}$. Actually, $\left\|T_{1}\right\| \leqslant c$ if $\left\|a_{1}\right\|_{0, R} \leqslant c_{1}$. Furthermore

$$
\left\|T_{2} f\right\|_{2, R} \leqslant c_{R}\left\|\nabla \cdot\left(a_{2} \nabla f\right)\right\|_{0, R} \leqslant c_{R} \cdot c_{2}\|f\|_{2, R} .
$$

Therefore $\left\|T_{2}\right\|<1$ if $c_{R} c_{2}<1$. Let us summarise the result. 
Proposition 1. If (3.48) holds and $q \equiv \omega^{2} c_{1} c_{R}+c_{2} c_{R}<1$ then the Born series $\sum_{n=0}^{\infty}\left(\omega^{2} T_{1}+T_{2}\right)^{n} f$ converges in $H_{R}^{2}$ and is majorised by the series $\sum_{n=0}^{\infty} q^{n}$.

\section{5}

Consider the inverse scattering problem for equation (3.7) with the scattering amplitude as the data. If the incident field is the plane wave $u_{0}=\exp \left(i \omega s_{0} \cdot x\right)$, then the scattering amplitude is given by

$$
f\left(s_{0}, s, \omega\right)=-\frac{1}{4 \pi} \int \exp (-\mathrm{i} \omega s \cdot y)\left[a_{1}(y) u(y) \omega^{2}+\nabla \cdot\left(a_{2}(y) \nabla u\right)\right] \mathrm{d} y .
$$

In the Born approximation $u=u_{0}$, and, integrating the second term in (52) by parts, one obtains for the scattering amplitude

$$
f=f_{\mathrm{B}}\left(s_{0}, s, \omega\right)=-\frac{\omega^{2}}{4 \pi} \int \exp \left[i \omega\left(s_{0}-s\right) \cdot y\right]\left(a_{1}(y)-s_{0} \cdot s a_{2}(y)\right) \mathrm{d} y .
$$

Consider $D=-4 \pi \omega^{-2} f_{\mathrm{B}}$ as the data. Assume that the data are known at $\omega=\omega_{j}$, $j=1,2, \omega_{1}<\omega_{2}$. Then the Fourier transforms $\tilde{\alpha}_{j}$ of $a_{j}(y), j=1,2$, can be found from (3.53). Indeed, set

$$
\omega\left(s_{0}-s\right)=\omega p, \text { where }|p|^{2}=2-2 \gamma \quad \text { and } \quad \gamma=s \cdot s_{0}=1-\frac{1}{2}|p|^{2} .
$$

Then

$$
\begin{aligned}
& D_{1}=\tilde{a}_{1}\left(\omega_{1} p\right)-\left(1-\frac{1}{2}|p|^{2}\right) \tilde{a}_{2}\left(\omega_{1} p\right) \\
& D_{2}=\tilde{a}_{1}\left(\omega_{2} p\right)-\left(1-\frac{1}{2}|p|^{2}\right) \tilde{a}_{2}\left(\omega_{2} p\right) .
\end{aligned}
$$

Set $q=\omega_{1} p$. Then (3.54) and (3.55) can be written as

$$
\begin{aligned}
& D_{1}(q)=\tilde{a}_{1}(q)-\left(1-|q|^{2} / 2 \omega_{1}^{2}\right) \tilde{a}_{2}(q) \\
& D_{2}\left(\omega_{1} q / \omega_{2}\right)=\tilde{a}_{1}(q)-\left(1-|q|^{2} / 2 \omega_{2}^{2}\right) \tilde{a}_{2}(q) .
\end{aligned}
$$

From (3.56) and (3.57) one finds $\tilde{a}_{j}(q)$ and, by applying the Fourier inversion, $a_{j}(y)$, $j=1,2$. Again the problem formulated at the end of $\S 3.2$ arises.

\section{An inversion formula in scattering theory}

\section{1}

In this section we solve a three-dimensional inverse scattering problem in the Born approximation, and the problem in $\S 3.2$.

Let

$$
\begin{array}{ll}
\left(\nabla^{2}+k^{2}-q(x)\right) u=0 & \text { in } R^{3} \\
u=\exp (\mathrm{i} k s \cdot x)+v & \\
v \sim \frac{\exp (\mathrm{i} k r)}{r} f\left(s, s^{\prime}, k\right) \quad \text { as } r=|x| \rightarrow \infty, x r^{-1}=s^{\prime} .
\end{array}
$$


The integral equation for $u$ is

$$
u=\exp (\mathrm{i} k s \cdot x)-\int g q u \mathrm{~d} y \quad g=\frac{\exp (\mathrm{i} k|x-y|)}{4 \pi|x-y|} .
$$

Therefore

$$
f\left(s, s^{\prime}, k\right)=-\frac{1}{4 \pi} \int \exp \left(-\mathrm{i} k s^{\prime} \cdot y\right) q u \mathrm{~d} y .
$$

In the Born approximation $(u=\exp (i k s \cdot x))$ one has

$$
f=f_{\mathrm{B}}=-\frac{1}{4 \pi} \int \exp \left[\mathrm{i} k\left(s-s^{\prime}\right) \cdot y\right] q(y) \mathrm{d} y .
$$

Let us assume that

$$
q(x) \in L^{2}\left(B_{R}\right) \quad q(x)=0 \quad \text { if }|x|>R .
$$

Then one obtains for $q(y)$ the integral equation

$$
\int_{|y| \leqslant R} \exp \left[-\mathrm{i} k\left(s^{\prime}-s\right) \cdot y\right] q(y) \mathrm{d} y=F\left[k\left(s^{\prime}-s\right)\right]
$$

where $F\left[k\left(s^{\prime}-s\right)\right]=-4 \pi f_{\mathrm{B}}$. In (4.8) $k>0$ is fixed and $s$ and $s^{\prime}$ run through the unit sphere $S^{2}$. Let $p=k\left(s^{\prime}-s\right)$. When $s$ and $s^{\prime}$ run through $S^{2}$ the vector $p$ runs through the ball $B_{2 k}=\{p:|p| \leqslant 2 k\}$. Equation (4.8) can be written as

$$
\int_{|y| \leqslant R} \exp (-\mathrm{i} p \cdot y) q(y) \mathrm{d} y=F(p) \quad|p| \leqslant 2 k .
$$

This equation is solvable in the class of functions $q$ satisfying conditions (4.7) iff $F(p) \in W_{R}$, where $W_{R}$ is the set of entire functions of exponential type $\leqslant R$ square integrable over $R^{3}$, that is of entire functions such that

$$
|F(p)| \leqslant c \exp (R|p|) \quad \int_{R^{j}}|F(p)|^{2} \mathrm{~d} p<\infty
$$

This is a variant of the Paley-Wiener theorem. If

$$
\tilde{q}(p) \equiv \int \exp (-\mathrm{i} p \cdot y) q \mathrm{~d} y=0 \quad \text { for }|p|>2 k_{0}
$$

then one can find $q(y)$, the solution of equation (4.9), by taking the inverse Fourier transform of the function $F(p)$. But (4.11) is equivalent to the assumption that $q(1)$ is an entire function of class $W_{2 k_{0}}$ which is not the case in applications where assumptions (4.7) are more realistic. The idea of what follows is to approximate $q(x)$ satisfying conditions (4.7) by an entire function $q_{N}(x) \in W_{2 k_{0}}$ with arbitrary accuracy on $B_{R}:\left\|q_{N}-q\right\|_{R} \leqslant \varepsilon(N) \rightarrow 0, N \rightarrow \infty$. Here $\|\cdot\|_{R}$ is the norm in $L^{2}\left(B_{R}\right)$ or $C\left(B_{R}\right)$ depending on whether $q \in L^{2}\left(B_{R}\right)$ or $C\left(B_{R}\right)$. The function $q_{N}(x)$ will be explicitly constructed analytically from the data. 
4.2

The data in (4.8) are given on $S^{2} \times S^{2}$ and it is useful to have a formula which transforms the three-dimensional Fourier integral into an integral over $S^{2} \times S^{2}$. Such a formula in $R^{d}$, $d \geqslant 2$, is known (Devaney 1982, $d=2$; Beylkin 1983, $d \geqslant 2$ ):

$$
\begin{array}{rl}
(2 \pi)^{-d} \int_{|p| \leqslant 2 k} & F(p) \exp (\mathrm{i} p \cdot x) \mathrm{d} p \\
= & \frac{k^{d}}{8 \pi^{d} \omega_{d-1}} \int_{s^{d-1}} \int_{S^{d-1}} \frac{\left|s-s^{\prime}\right| \exp \left[\mathrm{i} k\left(s-s^{\prime}\right) \cdot x\right] F\left[k\left(s-s^{\prime}\right)\right] \mathrm{d} s \mathrm{~d} s^{\prime}}{\left(4-\left|s-s^{\prime}\right|^{2}\right)^{(d-3) / 2}}
\end{array}
$$

where $d s$ is the element of the area of the unit sphere $S^{d-1}$. The identity (4.12) holds for any $F \in L^{2}\left(B_{2 k}\right), S^{d-1}$ is the unit sphere in $R^{d}, \omega_{d}$ is the area of $S^{d-1}, \omega_{d}=2 \pi^{d / 2} / \Gamma(d / 2)$, where $\Gamma(x)$ is the gamma function. For $d=3$ one has

$$
\begin{aligned}
& (2 \pi)^{-3} \int_{|p| \leqslant 2 k} F(p) \exp (\mathrm{i} p \cdot x) \mathrm{d} p \\
& \quad=\frac{k^{3}}{(2 \pi)^{4}} \int_{s^{2}} \int_{s^{2}}\left|s-s^{\prime}\right| \exp \left[\mathrm{i} k\left(s-s^{\prime}\right) \cdot x\right] F\left[k\left(s-s^{\prime}\right)\right] \mathrm{d} s \mathrm{~d} s^{\prime} .
\end{aligned}
$$

For $d=2$ one has

$$
\begin{array}{rl}
(2 \pi)^{-2} \int_{|p| \leqslant 2 k} & F(p) \exp (\mathrm{i} p \cdot x) \mathrm{d} p \\
= & \frac{k^{2}}{16 \pi^{2}} \int_{s^{1}} \int_{s^{1}}\left|s-s^{\prime}\right|\left(4-\left|s-s^{\prime}\right|^{2}\right)^{1 / 2} \\
& \quad \times \exp \left[\mathrm{i} k\left(s-s^{\prime}\right) \cdot x\right] F\left[k\left(s-s^{\prime}\right)\right] \mathrm{d} s \mathrm{~d} s^{\prime} .
\end{array}
$$

If (4.11) holds then (4.8) is solvable by the formula

$$
\begin{aligned}
q(x)=(2 \pi)^{-3} & \int \exp (\mathrm{i} p \cdot x) F(p) \mathrm{d} p \\
& =\frac{k^{3}}{(2 \pi)^{4}} \int_{s^{2}} \int_{S^{2}}\left|s-s^{\prime}\right| \exp \left[\mathrm{i} k\left(s-s^{\prime}\right) \cdot x\right] F\left[k\left(s-s^{\prime}\right)\right] \mathrm{d} s \mathrm{~d} s^{\prime}
\end{aligned}
$$

where formula (4.13) was used.

Before we pass to the formula for $q_{N}$ let us prove the following two lemmas.

Lemma 1. Equation (4.8) has no more than one solution.

Lemma 2. Formula (4.12) holds.

Proof of lemma 1. Equation (4.8) is equivalent to (4.9). If $F(p)=0,|p| \leqslant 2 k$ then

$$
\int_{|y| \leqslant R} \exp (-\mathrm{i} p \cdot y) q(y) \mathrm{d} y=0 \quad p \in R^{3} .
$$

Indeed, the left-hand side of (4.9) is an entire function of $p$. If this function vanishes in the ball $B_{2 k}$ it vanishes everywhere. From (4.16) one concludes that $q=0$. 
Proof of lemma 2. In this proof we use the identity for the spherical means (see John 1955, p 81). Let us define the spherical mean for a continuous function $F$ at the point $x$ :

$$
I(x, r)=\frac{1}{\omega_{d}} \int_{s^{d-1}} F(x+r s) \mathrm{d} s .
$$

Clearly, if $F$ is continuous, then

$$
I(x, 0)=F(x) \text {. }
$$

Let us define the iterated spherical mean

$$
M(x, \lambda, \mu)=\frac{1}{\omega_{d}^{2}} \int_{S^{d-1}} \int_{s^{d-1}} F\left(x+\lambda s+\mu s^{\prime}\right) \mathrm{d} s \mathrm{~d} s^{\prime} .
$$

One has $M(x, \lambda, \mu)=M(x, \mu, \lambda), M(x, \lambda, 0)=M(x, 0, \lambda)=I(x, \lambda), M(x, 0,0)=F(x)$. The identity which we need is of the form $(\lambda, \mu>0)$ :

$$
\begin{aligned}
M(x, \lambda, \mu)= & \frac{2 \omega_{d-1}}{\omega_{d}(2 \lambda \mu)^{d-2}} \\
& \quad \times \int_{\mu-\lambda}^{\mu+\lambda}[(r+\mu-\lambda)(r+\mu+\lambda)(r-\mu+\lambda)(\lambda-r+\mu)]^{(d-3) / 2} r I(x, r) \mathrm{d} r .
\end{aligned}
$$

Set $\lambda=\mu=k$ in (4.20) to obtain

$$
M(x, k, k)=\frac{\omega_{d-1}}{\omega_{d} k^{2 d-4} 2^{d-3}} \int_{0}^{2 k}\left(4 k^{2}-r^{2}\right)^{(d-3) / 2} r^{d-2} I(x, r) \mathrm{d} r .
$$

Let $h_{y}(p)=|p|\left(4 k^{2}-|p|^{2}\right)^{-(d-3) / 2} \hat{F}(p) \exp (\mathrm{i} p \cdot y)$, where $\hat{F}(p)$ is a continuous function vanishing outside $B_{2 k}$. Taking the spherical mean of $h_{y}(p)$ yields

$$
I_{y}(0, r)=\frac{1}{\omega_{d}} \frac{r}{\left(4 k^{2}-r^{2}\right)^{(d-3) / 2}} \int_{s^{d-1}} \hat{F}(r s) \exp (\mathrm{i} r s \cdot y) \mathrm{d} s .
$$

In formula (4.21), the iterated spherical mean of $h_{y}(p)$ is

$$
\begin{aligned}
M(0, k, k) & =\frac{\omega_{d-1}}{\omega_{d}^{2} k^{2 d-4} 2^{d-3}} \int_{0}^{2 k} \mathrm{~d} r r^{d-1} \int_{S^{d-1}} \hat{F}(r s) \exp (\mathrm{i} r s \cdot y) \mathrm{d} s \\
& =\frac{\omega_{d-1}(2 \pi)^{d}}{\omega_{d}^{2} k^{2 d-4} 2^{d-3}} \frac{1}{(2 \pi)^{d}} \int_{B_{2 k}} \mathrm{~d} p \exp (\mathrm{i} p \cdot y) \hat{F}(p) .
\end{aligned}
$$

Let

$$
\begin{aligned}
& F(y)=\frac{1}{(2 \pi)^{d}} \int_{B_{2 k}} \mathrm{~d} p \exp (\mathrm{i} p \cdot y) \hat{F}(p) \mathrm{d} p \\
& \hat{F}(p)=\int_{R^{3}} \mathrm{~d} y \exp (-\mathrm{i} p \cdot y) F(y) .
\end{aligned}
$$

Let us write $M(0, k, k)$ for the function $h_{y}(p)$ using definition (4.19) and then equate the 
expression to (4.23). This will lead to (4.15). Take $F(p)=h_{y}(p)$ in (4.19) to obtain

$$
\begin{aligned}
M(0, k, k)= & \frac{1}{\omega_{d}^{2}} \int_{S^{d-1}} \int_{S^{d-1}}\left|k\left(s+s^{\prime}\right)\right|\left(4 k^{2}-k^{2}\left|s+s^{\prime}\right|^{2}\right)^{-(d-3) / 2} \hat{F}\left[k\left(s+s^{\prime}\right)\right] \\
& \times \exp \left[\mathrm{i} k\left(s+s^{\prime}\right) \cdot y\right] \mathrm{d} s \mathrm{~d} s^{\prime} .
\end{aligned}
$$

Compare (4.25) and (4.23) to obtain

$$
\begin{gathered}
\frac{1}{(2 \pi)^{d}} \int_{B_{2 k}} \mathrm{~d} p \exp (\mathrm{i} p \cdot y) \hat{F}(p)=\frac{k^{d}}{8 \pi^{d} \omega_{d-1}} \int_{s^{d-1}} \int_{s^{d-1}}\left(4-\left|s+s^{\prime}\right|^{2}\right)^{-(d-3) / 2} \\
\times\left|s+s^{\prime}\right| \hat{F}\left[k\left(s+s^{\prime}\right)\right] \exp \left[\mathrm{i} k\left(s+s^{\prime}\right) \cdot y\right] \mathrm{d} s \mathrm{~d} s^{\prime} .
\end{gathered}
$$

If one changes $s^{\prime}$ to $-s^{\prime}$ in (4.26) then one obtains (4.12).

\section{3}

Let us proceed to the construction of $q_{N}(x)$ which approximates the solution $q(y)$ of equation (4.8). We assume that the data $F\left[k\left(s^{\prime}-s\right)\right]$ are exact, so that equation (4.8) is solvable. The calculations are given for the general case of $d$-dimensional space.

Let us multiply (4.8) by $\exp \left[i k\left(s^{\prime}-s\right) \cdot x\right] h_{N}\left[k\left(s^{\prime}-s\right)\right]$ and integrate over $S^{d-1} \times S^{d-1}$ to obtain

$$
\begin{aligned}
\int_{|y| \leqslant R} \mathrm{~d} y q(y) & \int_{S^{d-1}} \int_{s^{d-1}} \exp \left[\mathrm{i} k\left(s^{\prime}-s\right) \cdot(x-y)\right] h_{N}\left[k\left(s^{\prime}-s\right)\right] \mathrm{d} s \mathrm{~d} s^{\prime} \\
& =\int_{s^{d-1}} \int_{s^{d-1}} F\left[k\left(s^{\prime}-s\right)\right] h_{N}\left[k\left(s^{\prime}-s\right)\right] \exp \left[\mathrm{i} k\left(s^{\prime}-s\right) \cdot x\right] \mathrm{d} s \mathrm{~d} s^{\prime} .
\end{aligned}
$$

Here $h_{N}(p)$ is some function which will be specified later.

Our problem will be solved if the sequence defined by the formula

$$
\delta_{N}(x)=\int_{S^{i-1}} \int_{S^{d-1}} \exp \left[\mathrm{i} k\left(s^{\prime}-s\right) \cdot x\right] h_{N}\left[k\left(s^{\prime}-s\right)\right] \mathrm{d} s \mathrm{~d} s^{\prime}
$$

is a deita sequence in $L^{2}\left(B_{R}\right)$ or $C\left(B_{R}\right)$ in the sense that

$$
\left\|q(x)-q_{N}(x)\right\|_{R} \equiv \varepsilon(N) \rightarrow 0 \quad \text { as } N \rightarrow \infty .
$$

Here

$$
q_{N}(x)=\int_{|y| \leqslant R} \delta_{N}(x-y) q(y) \mathrm{d} y
$$

and the norm $\|\cdot\|_{R}$ in (4.29) is the $L^{2}\left(B_{R}\right)\left(C\left(B_{R}\right)\right)$ norm if $q(x) \in L^{2}\left(B_{R}\right)\left(q(x) \in C\left(B_{R}\right)\right)$. One can estimate $\varepsilon(N)$ if some additional information about $q(x)$ is known, e.g. bounds $|q| \leqslant a,|\nabla q| \leqslant b$.

Let us choose $h_{N}$ such that the sequence (4.28) becomes a delta sequence in the sense defined above. It follows from (4.12) that

$$
\begin{aligned}
\int_{s^{d-1}} \int_{s^{d-1}} \exp \left[i k\left(s^{\prime}-s\right) \cdot x\right] h_{N}\left[k\left(s^{\prime}-s\right)\right] \mathrm{d} s \mathrm{~d} s^{\prime} \\
=\frac{8 \pi^{d} \omega_{d-1}}{k^{2 d-4}(2 \pi)^{d}} \int_{B_{2 k}} \mathrm{~d} p \exp (\mathrm{i} p \cdot x)\left(4 k^{2}-|p|^{2}\right)^{(d-3) / 2}|p|^{-1} h_{N}(p) .
\end{aligned}
$$


Let us denote

$$
h_{N}(p)|p|^{-1}\left(4 k^{2}-|p|^{2}\right)^{(d-3) / 2} \frac{8 \omega_{d-1} \pi^{d}}{k^{2 d-4}}=A_{N}(p) .
$$

Then, comparing (4.28), (4.31) and (4.32) yields

$$
\delta_{N}(x)=\frac{1}{(2 \pi)^{d}} \int_{B_{2 k}} \mathrm{~d} p \exp (\mathrm{i} p \cdot x) A_{N}(p) .
$$

Therefore we need to construct a delta sequence in $L^{2}\left(B_{R}\right)$ of functions $\delta_{N} \in W_{2 k}$. Such a sequence was constructed by $\operatorname{Ramm}(1980)$ :

$\delta_{N}(x)=\left(\frac{1}{\left|B_{2 k}\right|} \int_{|p| \leqslant 2 k} \mathrm{~d} p \exp \left(\frac{\mathrm{i} p \cdot x}{2 N+d}\right)\right)^{2 N+d}\left(1-\frac{|x|^{2}}{4 R^{2}}\right)^{N}\left(\frac{N}{4 \pi R^{2}}\right)^{d / 2}$.

Here $\left|B_{k}\right|$ is the volume of the ball of radius $k,\left|B_{k}\right|=k^{d} \pi^{d / 2} / \Gamma\left[\frac{1}{2}(d+2)\right]$ and

$$
\int_{|p|<2 k} \mathrm{~d} p \exp \left(\frac{\mathrm{i} p \cdot x}{2 N+d}\right)=\frac{(2 k)^{d / 2}(2 N+d)^{d / 2}(2 \pi)^{d / 2}}{|x|^{d / 2}} J_{d / 2}\left(\frac{2 k|x|}{2 N+d}\right)
$$

where $J_{m}(x)$ is a Bessel function. Thus

$\delta_{N}(x)=\left(1-\frac{|x|^{2}}{4 R^{2}}\right)^{N}\left(\frac{N}{4 \pi R^{2}}\right)^{d / 2}\left(2^{d / 2} \Gamma\left(\frac{1}{2} d+1\right) \frac{J_{d / 2}[2 k|x| /(2 N+d)]}{[2 k x /(2 N+d)]^{d / 2}}\right)^{2 N+d}$

where $\Gamma(x)$ is the gamma function.

In particular, for $d=3$ one has

$$
\delta_{N}(x)=\left(1-\frac{|x|^{2}}{4 R^{2}}\right)^{N}\left(\frac{N}{4 \pi R^{2}}\right)^{3 / 2}\left(\frac{\sin \lambda-\lambda \cos \lambda}{\lambda^{3} / 3}\right)^{2 N+3} \quad \lambda=\frac{2 k|x|}{2 N+3} .
$$

Notice that the sequence (4.34) is a delta sequence on $L^{2}\left(B_{R}\right)$ but not on $L^{2}\left(R^{d}\right)$. One cannot construct a delta sequence $\delta_{N} \in W_{2 k}$ on $L^{2}\left(\boldsymbol{R}^{d}\right)$ because the Fourier transform $\hat{\delta}_{N}(p)$ of a function $\delta_{N} \in W_{2 k}$ should have its support in the ball $B_{2 k}$ while the support of the Fourier transform of a delta sequence on $L^{2}\left(\boldsymbol{R}^{d}\right)$ cannot stay within a fixed compact as $N \rightarrow \infty$. It follows from (4.32) and (4.33) that

$$
A_{N}(p)=\int_{R^{d}} \delta_{N}(x) \exp (-\mathrm{i} p \cdot x) \mathrm{d} x
$$

and

$$
h_{N}(p)=|p|\left(4 k^{2}-|p|^{2}\right)^{-(d-3) / 2} A_{N}(p) k^{2 d-4}\left(8 \pi^{d} \omega_{d-1}\right)^{-1} .
$$

It follows from (4.27), (4.28) and (4.30) that

$q_{N}(x)=\int_{s^{d-1}} \int_{S^{d-1}} F\left[k\left(s^{\prime}-s\right)\right] h_{N}\left[k\left(s^{\prime}-s\right)\right] \exp \left[\mathrm{i} k\left(s^{\prime}-s\right) \cdot x\right] \mathrm{d} s \mathrm{~d} s^{\prime}$.

Let us formulate the result.

Theorem 1. If equation (4.8) is solvable then formula (4.40) gives an approximate analytical solution to equation (4.8) in the sense that equation (4.29) holds. The sequence $h_{N}(x)$ in (4.40) is given by formulae (4.39), (4.38) and (4.36). 
Remark 1. If

$$
|q| \leqslant a \quad \text { and } \quad|\nabla q| \leqslant b
$$

then, in the norm of $C\left(B_{R}\right)$, the estimate for $\varepsilon(N)$ in (4.29) is of the form

$$
\varepsilon(N) \leqslant \Gamma\left[\frac{1}{2}(d+1)\right]\left(\Gamma\left(\frac{1}{2} d\right)\right)^{-1} b R N^{-1 / 2}+\mathrm{O}\left(N^{-1}\right) \quad N \rightarrow \infty .
$$

This is proved by Ramm (1980, p 211).

\section{4}

Let $d=3$. Suppose that the data are given with some error $\eta$, so that $F_{\eta}\left(k\left|s^{\prime}-s\right|\right)$ is known such that $\left|F-F_{\eta}\right|<\eta$.

If one applies the inversion formula (4.40) to the noisy data $F_{\eta}$ then one obtains a function $q_{N \eta}$ such that

$$
\left\|q_{N, \eta}-q\right\|_{R} \leqslant\left\|q_{N \eta}-q_{N}\right\|_{R}+\left\|q_{N}-q\right\|_{R} \leqslant \eta b_{N}+\varepsilon(N)
$$

where the norm is taken in $C\left(B_{R}\right) ; \varepsilon(N)$, given by (4.29), is the error of the inversion formula (4.40) in the absence of noise, and

$$
b_{N}=\int_{s^{2}} \int_{s^{2}}\left|h_{N}\left[k\left(s^{\prime}-s\right)\right]\right| \mathrm{d} s \mathrm{~d} s^{\prime}
$$

where, for $d=3$, from (4.39) one has $h_{N}(p)=|p| k^{2} A_{N}(p)\left(16 \pi^{4}\right)^{-1}, A_{N}(p)$ is given by (4.38) and $\delta_{N}$ is given by (4.37). For a fixed $\eta>0$ one can find $N=N(\eta)$ for which

$$
\alpha(N, \eta) \equiv \eta b_{N}+\varepsilon(N)=\min
$$

Such an $N(\eta)$ does exist because $\varepsilon(N) \rightarrow 0$ and $b_{N} \rightarrow \infty$ as $N \rightarrow \infty$. For this $N(\eta)$ the error $\alpha(\eta) \equiv \alpha(N(\eta), \eta) \rightarrow 0$ as $\eta \rightarrow 0$. Therefore

$$
\left\|q_{N(\eta), \eta}(x)-q(x)\right\|_{R} \rightarrow 0 \quad \text { as } \eta \rightarrow 0
$$

so that $q_{N(\eta), \eta}(x)$ is a stable approximation of $q(x)$ on $B_{R}$.

The following lemma is useful for computing $b_{N}$.

Lemma 3.

$\int_{s^{d-1}} \int_{S^{d-1}} h\left[k\left(s^{\prime}-s\right)\right] d s \mathrm{~d} s^{\prime}=\frac{\omega_{d-1}}{2^{d-3} k^{2 d-4}} \int_{|p| \leqslant 2 k} \mathrm{~d} p h(p)|p|^{-1}\left(4 k^{2}-|p|^{2}\right)^{-(d-3) / 2}$.

Proof. The proof is similar to the proof of lemma 2 and therefore is left to the reader.

From (4.44) and (4.47) it follows that

$$
b_{N}=\frac{2 \pi}{k^{2}} \int_{|p| \leqslant 2 k} \mathrm{~d} p\left|h_{N}(p)\right||p|^{-1}=\frac{1}{8 \pi^{3}} \int_{|p| \leqslant 2 k}\left|A_{N}(p)\right| \mathrm{d} p .
$$

A rough estimate for $b_{N}$, which follows from (4.48), is

$$
b_{N} \leqslant \frac{\left|B_{2 k}\right|}{8 \pi^{3}} \max _{|p| \leqslant 2 k}\left|A_{N}(p)\right| \leqslant \frac{4 k^{3}}{3 \pi^{2}} \int_{R^{3}}\left|\delta_{N}(x)\right| \mathrm{d} x .
$$

Another estimate for $b_{N}$ follows directly from (4.44):

$$
b_{N} \leqslant(4 \pi)^{2} \max _{|p| \leqslant 2 k}\left|h_{N}(p)\right| \text {. }
$$

Estimate (4.50) can also be obtained from the rough estimate of the first integral in (4.48). 


\section{A model inverse problem of induction logging}

\section{1}

Consider a medium whose properties we want to find from the knowledge of the field on the ray $x_{3}<0$. One can consider a borehole-a cylindrical hole in the medium; along the $x_{3}$ axis the source and receiver can be moved. They can either be kept at a fixed distance $d$, or their positions can vary arbitrarily. The problem is to recover the properties of the medium from the measurements recorded by the receiver.

In induction logging the medium is characterised by the dielectric and magnetic parameters $\varepsilon=1, \mu=$ constant, and by the conductivity $\sigma=\sigma_{0}+v(X)$, where $\sigma_{0}=$ constant and $v(X)$ is the inhomogeneous part of $\sigma$. The displacement currents $\partial \mathscr{L} / \partial t$ are negligible. The vector potential $A$ satisfies the equation $\left(\nabla^{2}+k^{2}\right) A=-j, k^{2}=i \omega \mu \sigma(X)$ and $j$ is the source current. Let $X=(\rho, \varphi, \zeta)$ be the cylindrical coordinates of the point $X$. In induction logging it is always assumed that $j$ is a current in a circular loop of wire. The loop is perpendicular to the $x_{3}$ axis, it has a small radius $a$, and its centre is at a point $(0,0, \zeta)$. The vector potential $A$ under these assumptions and under the additional assumption $v=v(\rho, z)$ has only one non-zero component, $A_{\varphi}$, in the cylindrical coordinates, $\nabla \cdot A=0, E=i \omega \mu A$, $H=\nabla \times A$.

We consider a model scalar acoustic problem in which the field $u$ satisfies the equation

$$
\nabla^{2} u+k^{2}(X) u=-\delta(X-Y) \quad X, Y \in R^{3} .
$$

$k^{2}(X)=\mathrm{i} \omega \sigma(x), \sigma(X)=\sigma_{0}+v(X), \sigma_{0}=\mathrm{constant}>0, \omega>0, v(X)=0$ if $x_{3}>0$ or $|X|>R$, where $R>0$ is a fixed (arbitrarily large) number, $v \in L^{2}, \operatorname{Im} k(X)>0, k_{0}=\left(i \omega \sigma_{0}\right)^{1 / 2}$. The integral equation for $u$ is

$$
u(X, Y, \omega)=g(X, Y)+\mathrm{i} \omega \int g(X, Z) v(Z) u(Z, Y) \mathrm{d} Z
$$

where

$$
g=\frac{\exp \left(i k_{0}|X-Y|\right)}{4 \pi|X-Y|}
$$

If $\omega$ is sufficiently small then the integral equation is uniquely solvable by iteration and $u$ is analytic in the variable $p=\omega^{1 / 2}$ in a neighbourhood of the point $p=0$.

Therefore the following limit exists:

$$
f(X, Y) \equiv \lim _{\omega \rightarrow 0} 16 \pi^{2} \frac{u-g}{i \omega}=\int \frac{v(Z) \mathrm{d} Z}{|X-Z||Y-Z|} .
$$

The function $f(X, Y)$ is the datum. Let $X=(0,0, x), Y=(0,0, y)$. Under various assumptions on $v(Z)$ we give inversion algorithms for recovering $v(Z)$ from the data $f(x, y)$ measured on the $x_{3}$ axis.

\subsection{Inversion in the one-dimensional case}

Assume that $v(Z)=v\left(z_{1}, z_{2}, z_{3}\right)=v\left(z_{3}\right),\left|z_{3}\right| \leqslant R, r \leqslant R$, and $v=0$ otherwise. Here $r=\left(z_{1}^{2}+z_{2}^{2}\right)^{1 / 2}$. Let $X=(0,0, x), Y=(0,0, y), z_{3}=\zeta, y=x-d, d=$ constant. Then (5.2) takes the form

$$
\int_{-\infty}^{\infty} d \zeta v(\zeta) h(x-\zeta)=\psi(x) \quad-\infty<x<\infty
$$


where

$$
\begin{aligned}
& \psi(x) \equiv(2 \pi)^{-1} f(x, x-d) \\
& h(x-\zeta) \equiv \int_{0}^{R} \frac{\mathrm{d} r r}{\left[(\zeta-x)^{2}+r^{2}\right]^{1 / 2}\left[(\zeta-x+d)^{2}+r^{2}\right]^{1 / 2}} .
\end{aligned}
$$

Equation (5.3) is of convolution type and can be solved by Fourier's transform. If the data are noisy, i.e. $\psi_{\delta}$ is known instead of $\psi,\left|\psi_{\delta}-\psi\right| \leqslant \delta$, then a regularising algorithm should be used for the numerical solution of (5.3). For example, one can solve (5.3) with $\psi_{\delta}$ on the right-hand side by the formula

$$
v_{\delta}(\zeta)=\int_{-\infty}^{\infty} \mathrm{d} \lambda \exp (\mathrm{i} \lambda \zeta) \tilde{\psi}_{\delta}(\lambda) \tilde{h}^{-1}(\lambda) \exp (-\alpha(\delta)|\lambda|)(2 \pi)^{-1}
$$

where $\alpha(\delta) \rightarrow 0$ and $\delta \alpha^{-1}(\delta) \rightarrow 0$ (see Ramm 1980, p 207 for details and for an optimal choice of $\alpha(\delta)$ ). One can also solve the Wiener-Hopf-type equation

$$
\int_{0}^{\infty} \mathrm{d} \zeta v(\zeta) h(x-\zeta)=\psi(x) \quad 0 \leqslant x<\infty
$$

which models the case when measurements are taken strictly inside the borehole.

The same method is valid for inversion of the data at a fixed frequency $\omega>0$ in the Born approximation. The function analogous to $h(x-\zeta)$ in $(5.4)$ will be

$$
h_{\omega}(x-\zeta)=\int \mathrm{d} r r \exp (i \omega \beta) \alpha^{-1}
$$

where

$$
\alpha \equiv\left[(\zeta-x)^{2}+r^{2}\right]^{1 / 2}\left[(\zeta-x+d)^{2}+r^{2}\right]^{1 / 2} \equiv \alpha_{1} a_{2} \quad \beta=\alpha_{1}+\alpha_{2} .
$$

\subsection{The two-dimensional case}

5.3.1. The results of this section show that the data in the Born approximation

$$
F(X, Y) \equiv \frac{16 \pi^{2}(A-g)}{\mathrm{i} \omega \mu}=\int \frac{\exp \left[\mathrm{i} k_{0}(|X-Z|+|Z-Y|)\right] v(Z)}{|X-Z||Z-Y|} \mathrm{d} Z
$$

measured for all $X, Y$ belonging to the $\zeta$ axis and at a fixed frequency $\omega>0$ do determine $v(r, z)$ in some cases. Assume that $v=v(r, z)=0$ if $r \geqslant R$ or $|z| \geqslant R$.

Let us use the spheroidal coordinates as used by Ramm (1966):

$$
\begin{aligned}
& z_{3}=l s t+\frac{1}{2}\left(x_{3}+y_{3}\right) \quad z_{2}=l\left(s^{2}-1\right)^{1 / 2}\left(1-t^{2}\right)^{1 / 2} \cos \theta+\frac{1}{2}\left(x_{2}+y_{2}\right) \\
& z_{1}=l\left(s^{2}-1\right)^{1 / 2}\left(1-t^{2}\right)^{1 / 2} \sin \theta+\frac{1}{2}\left(x_{1}+y_{1}\right)
\end{aligned}
$$

where $l=\frac{1}{2}|X-Y|,|X-Z|+|Z-Y|=2 l s, 1 \leqslant s<\infty,|X-Z|-|Z-Y|=2 l t,-1 \leqslant t \leqslant 1$ and $\theta$ is the angle between the plane spanned by $X-Z$ and $Y-Z$ and a fixed plane containing $X$ and $Y$. The Jacobian of the transformation $Z \rightarrow(s, t, \theta)$ is $J=l^{3}\left(s^{2}-t^{2}\right)$. In our case $X$ and $Y$ belong to the $\zeta$ axis. As the fixed plane we take the plane $z_{1} z_{3}, z_{3}=\zeta$, so that $\theta$ is the angular variable in the cylindrical coordinate system. In the new variables (5.5) takes the form

$2 \pi l \int_{-1}^{\infty} \mathrm{d} s \exp \left(2 \mathrm{i} k_{0} l s\right) \int_{-1}^{\cdot 1} \mathrm{~d} t v\left[l\left(s^{2}-1\right)^{1 / 2}\left(1-t^{2}\right)^{1 / 2}, l s t+\frac{1}{2}(x+y)\right]=F(x . y)$ 
where $x$ and $y$ denote the $\zeta$ components of the vectors $X$ and $Y, \frac{1}{2}(x-y)=l$ if $x \geqslant y$. From (5.6) it is clear that under our assumption $v=v(r, z)$ the data depend on $m=\frac{1}{2}(x+y)$ and $l=\frac{1}{2}|x-y|$.

5.3.2. Let us assume that $F$ is given for all frequencies $\omega$, i.e. for all $k_{0}, k_{0}=$ $[(1+i) / \sqrt{2}] \sigma_{0}^{1 / 2} \omega^{1 / 2}$. Then the function

$$
p(s, l) \equiv \int_{-1}^{l} \mathrm{~d} t v\left[l\left(s^{2}-1\right)^{1 / 2}\left(1-t^{2}\right)^{1 / 2}, l s t+m\right]
$$

can be found from (5.6) by taking the inverse Fourier transform in $k_{0}$. Therefore one obtains the equation for $v(r, \zeta)$

$$
\int_{-1}^{1} v\left[l\left(s^{2}-1\right)^{1 / 2}\left(1-t^{2}\right)^{1 / 2}, l s t+m\right] \mathrm{d} t=p(s, l, m) \quad s \geqslant 1 \quad l \geqslant 0
$$

where $p(s, l, m)$ is known.

Let $\xi=l\left(s^{2}-1\right)^{1 / 2}\left(1-t^{2}\right)^{1 / 2}, \eta=l s t+m$. Consider the ellipse $E(s, l)$ on the plane $(\xi, \eta)$ given by the equation $\xi^{2}\left(s^{2}-1\right)^{-1}+(\eta-m)^{2} s^{-2}=l^{2}$. Equation (5.7) can be written as

$$
\begin{aligned}
& \int w(\xi, \eta) \mathrm{d} S=p(s, l, m) \quad s \geqslant 1, \quad l \geqslant 0 \\
& E(s, l)=\left\{\xi, \eta: \xi^{2}\left(s^{2}-1\right)^{-1}+(\eta-m)^{2} s^{-2}=l^{2}\right\} .
\end{aligned}
$$

Here $d S$ is the arc length of the ellipse,

$$
\begin{aligned}
& \mathrm{d} S=l\left(\frac{s^{2}-t^{2}}{1-t^{2}}\right)^{1 / 2} \mathrm{~d} t \\
& u(\xi, \eta)=v(\xi, \eta)\left(\frac{1-t^{2}}{s^{2}-t^{2}}\right)^{1 / 2} \frac{1}{l} \equiv v(\xi, \eta) a(\xi, \eta)
\end{aligned}
$$

and $a(\xi, \eta)$ is the function $\left[\left(1-t^{2}\right) /\left(s^{2}-t^{2}\right)\right]^{1 / 2} / l$ expressed in the variables $\xi, \eta, s^{2}+t^{2}=$ $\xi^{2} l^{-2}+(\eta-m)^{2} l^{-2}+1, s^{2} t^{2}=(\eta-m)^{2} l^{-2}$. Problem (5.8) is an integral geometry problem. If $w$ is found from (5.8) then $v$ is found since $a(\xi, \eta)$ is known. It seems that equation (5.8) was not studied in the literature. A similar equation in which the ellipses had one focus at the origin and the other running through one of the coordinate axes was studied by Lavrentiev et al $(1970,1980)$, but their results are not applicable to equation $(5.8)$ because the families of ellipses are different.

5.3.3. Consider the inversion scheme for the case when $v(r, z)=v(z)$. If $v=v(z)$ does not depend on $r$ then $(5.7)$ takes the form

$$
\int_{-1}^{1} \mathrm{~d} t v(l s t+m)=p(s, l, m) \quad s \geqslant 1, \quad l \geqslant 0 .
$$

Then $s=m / l$ and $m(t+1)=\tau$. Then (5.9) can be written as

$$
\int_{0}^{2 m} v(\tau) \mathrm{d} \tau=m p(m / l, l, m) \equiv h(m, l) \quad l \geqslant 0, \quad m \geqslant l .
$$

From (5.10) one finds by differentiation that

$$
v(2 m)=\frac{1}{2} \frac{\mathrm{d} h(m, l)}{\mathrm{d} m} \quad v(z)=\left.\frac{1}{2}\left(\frac{\mathrm{d} h}{\mathrm{~d} m}\right)\right|_{m=z / 2} \quad m \geqslant l .
$$


Thus, the inversion scheme in this subsection can be summarised as follows. First, take the inverse Fourier transform of the data $F\left(x, y, k_{0}\right)$ in the variable $k_{0}$ (see equation (5.6)) to obtain (5.7). Then, take $s=m l^{-1}$ in (5.7) and find $v(z)$ by formula (5.11). In this scheme the data are assumed to be three-dimensional (a function of $l, m$ and $k_{0}$ ), while the conductivity is assumed to be a function of $z$ only, i.e. a function of one variable. The differentiation in (5.11) makes the inversion an ill-posed problem. Ramm $(1981,1984)$ has given an algorithm for stable differentiation of noisy data. There is considerable freedom in the choice of the method for solving (5.9). For example, one could set $m=l$ and $s=1$ and solve (5.9) as in $\S 5.3 .6$ below.

5.3.4. Consider the two-dimensional inhomogeneity $v(r, z)$ and assume that the dependence on $r$ is linear near the borehole, that is $v(r, z)=v(0, z)+(\partial v(0, z) / \partial r) r \equiv v(z)+w(z) r$. Then (5.7) can be written as

$$
\begin{gathered}
\int_{-1}^{1} v(l s t+m) \mathrm{d} t+l \int_{-1}^{1} w(l s t+m)\left(1-t^{2}\right)^{1 / 2} \mathrm{~d} t\left(s^{2}-1\right)^{1 / 2} \\
=p(s, l, m) \quad s \geqslant 1, \quad l \geqslant 0 .
\end{gathered}
$$

Take $s=1$ in (5.12) and solve (5.12) for $v$. (See $\S 5.3 .6$ below.) If $v(z)$ is known then (5.12) can be written as an equation for $w$ :

$$
\begin{aligned}
& \int_{-1}^{1} w(l s t+m)\left(1-t^{2}\right)^{1 / 2} \mathrm{~d} t=p_{1}(s, l, m) \\
& p_{1} \equiv\left(p(s, l, m)-\int_{-1}^{1} v(l s t+m) \mathrm{d} t\right)\left(s^{2}-1\right)^{-1 / 2} l^{-1} .
\end{aligned}
$$

Equation (5.13) can be solved for $w$. Notice that the function $p_{1}(s, l, m)$ is continuous as $s \rightarrow 1$ because of the choice of $v$. One has, if $m=l, p_{1}(1, l) \equiv p_{1}(1, l, l)$,

$$
\int_{0}^{2} w(l \tau)\left[1-(\tau-1)^{2}\right]^{1 / 2} \mathrm{~d} \tau=p_{1}(1, l) \quad l \geqslant 0 .
$$

Take the Mellin transform of (5.14) to obtain

$$
\hat{w}(u) a(u)=b(u) \quad \hat{w}(u)=b(u) a^{-1}(u)
$$

where $\hat{w}(u) \equiv \int_{0}^{\infty} w(x) x^{u-1} \mathrm{~d} x, b(u)=\hat{p}_{1}(1, l), a(u) \equiv \int_{0}^{2} \tau^{-u}\left[1-(\tau-1)^{2}\right]^{1 / 2} \mathrm{~d} \tau$, and the formula $\hat{w}(x \tau)=\hat{w}(u) \tau^{-u}$ was used. To find $w(z)$ take the inverse Mellin transform of $a^{-1}(u) b(u)$.

5.3.5. A general idea for inversion. Let $A=A_{0}+i \omega \mu \int g v A \mathrm{~d} Z$ be equation (5.1) corresponding to the source which produces potential $A_{0}$ in the absence of the inhomogeneity $v . A-A_{0} \equiv A^{\prime}$ is the scattered field. In the Born approximation one has $A^{\prime}=\mathrm{i} \omega \mu \int \mathrm{g} v A_{0} \mathrm{~d} Z$. One can try to find $A_{0}$ such that this equation can be solved for $v$.

5.3.6. A way to solve the equation

$$
\int_{-1}^{1} v(l t+m) \mathrm{d} t=p(1, l, m) \quad l \geqslant 0
$$


is to take $m=l$, set $l(t+1)=\tau$, write the equation as

$$
\int_{0}^{2 l} v(\tau) \mathrm{d} \tau=l p(1, l, l) \equiv h(l)
$$

and find

$$
v(2 l)=\frac{1}{2} \frac{\mathrm{d} h(l)}{\mathrm{d} l} \quad v(z)=\left.\frac{1}{2} \frac{\mathrm{d} h(l)}{\mathrm{d} l}\right|_{l=z / 2} .
$$

5.3.7. Assume $v=v(r)$. Then (5.7) becomes

$$
\int_{-1}^{1} v\left\{l\left[\left(s^{2}-1\right)\left(1-t^{2}\right)\right]^{1 / 2}\right\} \mathrm{d} t=p(s, l) \quad s \geqslant 1, \quad l \geqslant 0
$$

( $p$ does not depend on $m$ ). This equation can be solved analytically even in the case when $s$ (or $l$ ) is fixed. Indeed, let $l\left(s^{2}-1\right)^{1 / 2}=z$. Then $(5.16)$ becomes

$$
\int_{0}^{1} v\left[z\left(1-t^{2}\right)^{1 / 2}\right] \mathrm{d} t=f(z) \quad f(z) \equiv 0.5 p\left[s, z\left(s^{2}-1\right)^{-1 / 2}\right] \quad s>1 \text { if fixed. }
$$

Let $\left(1-t^{2}\right)^{1 / 2}=y, z y=x$. Then (5.17) becomes

$$
\int_{0}^{z} v(x) x\left(z^{2}-x^{2}\right)^{-1 / 2} \mathrm{~d} x=z f(z) \quad z \geqslant 0 .
$$

This equation can be solved by the formula

$$
v(z)=\frac{1}{z} \frac{2}{\pi} \frac{\mathrm{d}}{\mathrm{d} z} \int_{0}^{z} \frac{x^{2} f(x) \mathrm{d} x}{\left(z^{2}-x^{2}\right)^{1 / 2}}
$$

To derive (5.19) multiply (5.18) by $2 z\left(y^{2}-z^{2}\right)^{-1 / 2}$, integrate over $z$ in the range $(0, y)$, and use the formula $\int_{x}^{y} 2 z \mathrm{~d} z /\left[\left(y^{2}-z^{2}\right)\left(z^{2}-x^{2}\right)\right]^{1 / 2}=\pi$. This is a variant of Abel's equation.

5.3.8. Assume $v(r, z)=v_{0}(z)+v_{1}(z) h\left(r_{0}-r\right)$ where $v_{0}(z), v_{1}(z)$ are to be found, $r_{0}>0$ is to be found, $h(x)=1$ for $x \geqslant 0, h(x)=0$ for $x<0$. This model is used in geophysics. Then (5.7) becomes

$$
\int_{-1}^{1} v_{0}(l s t+m) \mathrm{d} t+\int_{-1}^{1} v_{1}(l s t+k m) h\left[r_{0}-l\left(s^{2}-1\right)^{1 / 2}\left(1-t^{2}\right)^{1 / 2}\right] \mathrm{d} t=p(s, l, m) .
$$

Set $s=1$ and find $v_{0}+v_{1}$ as in $\S 5.3 .6$. Take $l\left(s^{2}-1\right)^{1 / 2}$ large, then the second integral in $(5.20)$ is small and (5.20) reduces to (5.9) with $v=v_{0}$. Solve for $v_{0}$. Since $v_{0}+v_{1}$ and $v_{0}$ are found both functions $v_{0}$ and $v_{1}$ are found. Equation (5.20) can be written as

$$
\int_{1 \geqslant|t|>\left[1-r_{0}^{2} l^{-1}\left(s^{2}-1\right)^{-1}\right]^{1 / 2}} v_{1}(l s t+m) \mathrm{d} t=p_{1}(s, l, m) \equiv p(s, l, m)-\int_{-1}^{1} v_{0}(l s t+m) \mathrm{d} t
$$

where $p_{1}(s, l, m)$ is known. Let $l^{-2}\left(s^{2}-1\right)^{-1}=\beta$. Then (5.21) becomes

$$
V\left[m+l s\left(1-r_{0}^{2} \beta\right)^{1 / 2}\right]-V\left(m-l s\left(1-r_{0}^{2} \beta\right)^{1 / 2}\right]=p_{2}(s, \beta, m)
$$

where $p_{2}=-\left[p_{1}(s, l, m) l s-V(m+l s)+V(m-l s)\right]$ and $V$ is an antiderivative of $v_{1}, V^{\prime}=v_{1}$. 
Choose $l s\left(1-r_{0}^{2} \beta\right)^{1 / 2} \ll 1$. This is possible: $l s=l\left(1+l^{-2} \beta^{-1}\right)^{1 / 2}$ and one can take $l$ small and $\beta \sim r_{0}^{-2}$. Then Taylor's formula and (5.22) yield

$$
2 l s\left(1-r_{0}^{2} \beta\right)^{1 / 2} v_{1}(m)=p_{2}(s, \beta, m) \quad l^{-2}\left(s^{2}-1\right)^{-1}=\beta .
$$

Therefore

$r_{0}=\beta^{-1 / 2}\left[1-\left(\frac{p_{2}(s, \beta, m)}{2 l s v_{1}(m)}\right)^{2}\right]^{1 / 2} \quad l s\left(1-r_{0}^{2} \beta\right)^{1 / 2} \ll 1 \quad l^{-2}\left(s^{2}-1\right)^{-1}=\beta$.

Let us give an illustrative example of one of the inversion procedures. In this example all the computations are analytical.

Example. Assume that $v(X)=1$ if $r \leqslant 1,-1<x_{3}<0 v(X)=0$ otherwise, $\mu=\sigma_{0}=1$, and $d=0$, so that the positions of the source and the receiver are the same. The inversion procedure from $\S 5.2$ is based on equation (5.3). Under our assumptions this equation is

$2 \pi \int_{-\infty}^{\infty} \mathrm{d} z v(z) \int_{0}^{1} \frac{r \mathrm{~d} r}{(z-x)^{2}+r^{2}}=\pi \int_{-\infty}^{\infty} \mathrm{d} z v(z) \ln \left(1+\frac{1}{(z-x)^{2}}\right)=\psi(x)$.

Let $\tilde{\psi}(y)=\int_{-\infty}^{\infty} \psi(x) \exp (\mathrm{i} x y) \mathrm{d} x$. Taking the Fourier transform of (5.25) one obtains

$\pi \tilde{v}(y) \int_{-\infty}^{\infty} \exp (\mathrm{i} y x) \ln \left(1+\frac{1}{x^{2}}\right) \mathrm{d} x=\pi \tilde{v}(y) 2 \pi y^{-1}(1-\exp (-|y|))=\tilde{\psi}(y)$

where formula (1.5.10) of Erdelyi et al (1954) was used. Therefore

$$
\tilde{v}(y)=\tilde{y} \psi(y)\left[2 \pi^{2}(1-\exp (-|y|))\right]^{-1} .
$$

In particular, under the above assumptions about $v$ one has

$$
\begin{aligned}
& \psi(x)=\int_{-1}^{0} \mathrm{~d} z \ln \left(1+\frac{1}{(z-x)^{2}}\right) \\
& \tilde{\psi}(y)=\pi \frac{1-\exp (-\mathrm{i} y)}{\dot{\mathrm{i} y}} 2 \pi y^{-1}(1-\exp (-y)) .
\end{aligned}
$$

From (5.27) and the last formula one finds

$$
\begin{aligned}
& \tilde{v}(y)=\frac{1-\exp (-\mathrm{i} y)}{\mathrm{i} y} \\
& v(x)=(2 \pi)^{-1} \int_{-\infty}^{\infty} \exp (-\mathrm{i} x y) \tilde{v}(y) d y= \begin{cases}1 & -1<x<0 \\
0 & \text { otherwise. }\end{cases}
\end{aligned}
$$

Therefore the inhomogeneity is recovered from the exact data analytically. Suppose now that the data are noisy: $\psi_{\delta}$ is given instead of $\psi,\left\|\psi_{\delta}-\psi\right\| \leqslant \delta$, where $\delta>0$ is known, and $\|\psi\|=\left(\int_{-\infty}^{\infty}|\psi|^{2} \mathrm{~d} x\right)^{1 / 2}$. Let

$$
v_{\delta}(x)=(2 \pi)^{-1} \int_{-\infty}^{\infty} \exp (-\mathrm{i} y x) \tilde{\psi}_{\delta}(y) \frac{y \exp (-\alpha(\delta)|y|)}{2 \pi^{2}(1-\exp (-|y|))} \mathrm{d} y .
$$

We will show that under an appropriate choice of $\alpha(\delta)$ the function $v_{\delta}(x)$ gives a stable approximation to the solution $v(x)$ (corresponding to the exact data) in the sense that $\left\|v_{\delta}(x)-v(x)\right\| \rightarrow 0$ as $\delta \rightarrow 0$. The regularisation parameter $\alpha(\delta)$ will be chosen optimally. 
Let us assume that the unknown solution $v(x)$ satisfies the inequality $\int_{-\infty}^{\infty}|\tilde{v}|^{2}\left(1+y^{2}\right)^{b} \mathrm{~d} y \leqslant M^{2}$, where $b$ and $M$ are given positive constants. This assumption means that the solution lies in a certain compact set in $L^{2}$. Assume for simplicity that $b>\frac{3}{2}$. It will be clear soon how this assumption is used. Parseval's equality yields

$$
\begin{gathered}
\left\|v_{\delta}-v\right\|=(2 \pi)^{-1 / 2} \mid \tilde{v}_{\delta}-\tilde{v} \| \leqslant(2 \pi)^{-1 / 2}\left(\left\|\tilde{\psi}_{\delta}-\tilde{\psi}\right\| \cdot\left\|\frac{\exp (-\alpha|y|)|y|}{1-\exp (-|y|}\right\|\right. \\
\left.+\left\|(\tilde{\psi} / \tilde{h})\left(1+y^{2}\right)^{b / 2}\right\| \cdot\left\|\frac{1-\exp (-\alpha|y|)}{\left(1+y^{2}\right)^{b / 2}}\right\|\right) \\
\leqslant c\left(\delta \alpha^{-3 / 2}+M \alpha\right) \quad c=\text { constant }>0 .
\end{gathered}
$$

The estimate $\left\|(1-\exp (-\alpha|y|)) /\left(1+y^{2}\right)^{b / 2}\right\| \leqslant c \alpha$ holds if $b>\frac{3}{2}$. Let us find $\alpha=\alpha(\delta)$ such that $\delta \alpha^{-3 / 2}+M \alpha=\min$. One has $\alpha(\delta)=\left(\frac{2}{3} M / \delta\right)^{-2 / 5}=c_{1} \delta^{2 / 5}, c_{1}=\left(\frac{2}{3} M\right)^{-2 / 5}$. For this optimal $\alpha(\delta)$ the error of the approximate solution is $\left\|v_{\delta}-v\right\| \leqslant$ constant $\times \delta^{2 / 5}$, as $\delta \rightarrow 0$.

Coen and Yu (1981), Minerbo (1983) and Oristaglio (1983) give many references on the work done in induction logging theory.

\section{Acknowledgments}

The author thanks his colleagues Drs J Blackledge, P Martin, G Minerbo, M Oristaglio, $O L$ Weaver and A Weglein for discussions and collaboration.

\section{References}

Akhiezer N 1956 Theory of Approximation (New York: Ungar)

1965 The Classical Moment Problem and Some Related Questions in Analysis (Edinburgh: Oliver and Boyd)

Bellman R, Kalaba R and Lockett J 1966 Numerical Inversion of the Laplace Transform (New York: Elsevier) Beylkin $G 1983$ The fundamental identity for iterated spherical means and the inversion formula for diffraction tomography and inverse scattering J. Math. Phys. 24 1399-400

Blackledge J 1983 The theory of quantitative acoustic scatter imaging $P h D$ Thesis University of London Chadan K and Sabatier P 1977 Inverse Problems in Quanium Scaitering (New York: Springer)

Coen $S$ and $Y u M 1981$ The inverse problem of the direct current conductivity profile of a layered earth Geophysics $461702-13$

Davies B and Martin B 1979 Numerical inversion of the Laplace transform J. Comput. Phys. 33 1-32

Devaney A 1982 Inversion formula for inverse scattering within the Born approximation Opt. Lett. 7 111-2

Erdelyi A et al 1954 Tables of Integral Trantsforms (New York: McGraw-Hill)

Ewing Wet al 1957 Elastic Waves in Layered Media (New York: McGraw-Hill)

Gradshteyn I S and Ryzhik I M 1965 Tables of Integrals, Series and Products (New York: Academic)

Ivanov V K et al 1978 Theory of Linear Ill-posed Problems and Applications (Moscow: Nauka)

John F 1955 Plane Waves and Spherical Means (New York: Interscience)

Kantorovich L and Akilov G 1980 Functional Analvsis (New York: Pergamon)

Kristensson G, Ramm A G and Ström S 1983 Convergence of the T-matrix approaci in scattering theory II J. Math. Phvs. 24 2619-31

Krylov V and Skoblya N 1969 Handbook of Numerical Inversion of Laplace Transform (Jerusalem: Israel Sci. Transl.)

Lavrentiev M M et al 1970 Multidimensional inverse problems for differential equations Lecture Notes in Mathematics N167 (New York: Springer)

1980 Ill-Posed Problems of Mathematical Physics and Analysis (Moscow: Nauka) 
Lubarskii G 1957 Group Theory and its Applications in Physics (Moscow: Fizmatgiz)

Martin P A and Ramm A G 1985 Inverse scattering in geophysics III Proc. R. Soc. to be published

Minerbo G 1983 Radial inversion of TE measurements in a borehole Preprint

Oristaglio M 1983 Born inversion of the induction log Preprint

Ramm A G 1966 Domain free from resonances in the three-dimensional scattering problem Sov. Phys.-Dokl. 166 1319-22 (Math. Rev. 34 3902)

1980 Theory and Applications of Some New classes of Integral Equations (New York: Springer)

1981 Stable solutions of some ill-posed problems Math. Meth. Appl. Sci. 3 336-63

- 1982a Convergence of the $T$-matrix approach to scattering theory J. Math. Phys. 23 1123-5

— 1982b Convergence of the T-matrix approach in the potential scattering J. Math. Phys. 23 2408-9

1983a An inversion formula in scattering theory Phys. Lett. 99A 201-4

$1983 \mathrm{~b}$ Inverse scattering for geophysical problems Phys. Lett. 99A 258-60

1984 Estimates of the derivatives of random functions J. Math. Anal. Appl. $102244-50$

1985 Inverse scattering in geophysics 4 . Inversion of the induction logging measurements. Geophvs. Expl. submitted for publication

Ramm A G and Weglein A 1984 Inverse scattering for geophysical problems II. Inversion of acoustical data J. Math. Phys. $253231-4$

Tikhonov A and Arsenin V 1977 Solutions of Ill-posed Problems (Washington, DC: Winston) 